In: "Harmonic Analysis and Applications,"

A volume in honor of John J. Benedetto,

Birkhäuser, Boston, 2006, pp. 171--206.

\title{
Linear Independence of Finite Gabor Systems
}

\author{
Christopher Heil \\ School of Mathematics, Georgia Institute of Technology, Atlanta, GA 30332, USA \\ heil@math.gatech.edu
}

\begin{abstract}
Summary. This chapter is an introduction to an open conjecture in time-frequency analysis on the linear independence of a finite set of time-frequency shifts of a given $L^{2}$ function. Background and motivation for the conjecture are provided in the form of a survey of related ideas, results, and open problems in frames, Gabor systems, and other aspects of time-frequency analysis, especially those related to independence. The partial results that are known to hold for the conjecture are also presented and discussed.
\end{abstract}

\subsection{Introduction}

In 1987, John Benedetto introduced two of his young graduate students, David Walnut and myself, to a new mathematical research group that had been formed at the MITRE Corporation in McLean, Virginia. Later, as a postdoc at MIT, I met Jay Ramanathan ${ }^{1}$ and Pankaj Topiwala, ${ }^{2}$ then members of the main MITRE math research group in Bedford, Massachusetts. We began working together on problems in time-frequency analysis and wavelets. One direction followed a beautiful insight of Ramanathan's, applying Gabor frame expansions to derive boundedness and spectral results for pseudodifferential operators [70]. In another direction, we explored the basic structure of Gabor frames, which ultimately led us to make the following conjecture (sometimes called today the HRT Conjecture, the Linear Independence Conjecture for Time-Frequency Shifts, or the Zero Divisor Conjecture for the Heisenberg Group).

Conjecture 9.1. If $g \in L^{2}(\mathbf{R})$ is nonzero and $\left\{\left(\alpha_{k}, \beta_{k}\right)\right\}_{k=1}^{N}$ is any set of finitely many distinct points in $\mathbf{R}^{2}$, then $\left\{e^{2 \pi i \beta_{k} x} g\left(x-\alpha_{k}\right)\right\}_{k=1}^{N}$ is a linearly independent set of functions in $L^{2}(\mathbf{R})$.

\footnotetext{
${ }^{1}$ Currently: Professor, Eastern Michigan University, Ypsilanti, Michigan 48197. Email: ramanath@emunix.emich.edu

${ }^{2}$ Currently: Founder, FastVDO LLC, 7150 Riverwood Drive, Columbia, Maryland 21046. Email: pnt@fastvdo.com
} 
Despite the striking simplicity of the statement of this conjecture, it remains open today in the generality stated. This chapter provides some background and motivation for the conjecture in the form of a survey of related ideas, results, and open problems in frames, Gabor systems, and other aspects of time-frequency analysis, especially those related to independence. After a brief statement in Section 9.2 of some of the partial results that are known concerning the conjecture, Section 9.3 discusses some of the subtleties involved in the seemingly simple concepts of spanning and independence when dealing with infinite-dimensional spaces. In particular, this motivates the definition of frames. In Section 9.4 we specialize to the case of Gabor frames, whose elements consist of time-frequency shifts of a given function. We survey some topics in Gabor theory, including other open problems related to the BalianLow Theorem and to Nyquist-type density phenomena for Gabor systems. The reader whose primary interest is the HRT Conjecture can skim or skip the surveys in Sections 9.3 and 9.4 and turn directly to Section 9.5, which discusses some of the partial results that have been obtained, and some of the ideas behind their proofs.

Throughout, the discussion is intended to be accessible to graduate students who have a little background in real and functional analysis and some familiarity with Hilbert spaces, especially $L^{2}(\mathbf{R})$ and $L^{2}[0,1]$. A summary of basic theorems from functional analysis can be found in the first chapter of [64]. Extensive references are given throughout, both to research papers and to textbooks or survey articles. The choice of references is usually made based more on their utility as background or additional reading than for historical completeness.

\subsection{Statement of Partial Results}

Despite attacks by a number of groups, the only published papers specifically about the HRT Conjecture appear to be [69], [96], and [88]. ${ }^{3}$ These will be quickly summarized now, with more details presented in Section 9.5.

The HRT Conjecture was first made in the paper [69], and some partial results were obtained there, including the following.

(a) If a nonzero $g \in L^{2}(\mathbf{R})$ is compactly supported, or just supported on a half-line, then the independence conclusion holds for any value of $N$.

(b) If $g(x)=p(x) e^{-x^{2}}$ where $p$ is a nonzero polynomial, then the independence conclusion holds for any value of $N$.

(c) The independence conclusion holds for any nonzero $g \in L^{2}(\mathbf{R})$ if $N \leq 3$.

\footnotetext{
${ }^{3} \mathrm{~A}$ recent preprint by Radu Balan, "A noncommutative Wiener lemma and a faithful tracial state on Banach algebras of time-frequency shift operators," contains some new partial results.
} 
(d) If the independence conclusion holds for a particular $g \in L^{2}(\mathbf{R})$ and a particular choice of points $\left\{\left(\alpha_{k}, \beta_{k}\right)\right\}_{k=1}^{N}$, then there exists an $\varepsilon>0$ such that it also holds for any $h$ satisfying $\|g-h\|_{2}<\varepsilon$, using the same set of points.

(e) If the independence conclusion holds for one particular $g \in L^{2}(\mathbf{R})$ and particular choice of points $\left\{\left(\alpha_{k}, \beta_{k}\right)\right\}_{k=1}^{N}$, then there exists an $\varepsilon>0$ such that it also holds for that $g$ and any set of $N$ points in $\mathbf{R}^{2}$ within $\varepsilon$ of the original ones.

It is perhaps surprising that there are almost no partial results formulated in terms of smoothness or decay conditions on $g$. In particular, Conjecture 1 is open even if we impose the extra hypothesis that $g$ lies in the Schwartz class $\mathcal{S}(\mathbf{R})$.

The next partial advance was made by Linnell in [96]. He used $C^{*}$-algebra techniques to prove that if the points $\left\{\left(\alpha_{k}, \beta_{k}\right)\right\}_{k=1}^{N}$ are a subset of some translate of a full-rank lattice in $\mathbf{R}^{2}$, then the independence conclusion holds for any nonzero $g$ (such a lattice has the form $A\left(\mathbf{Z}^{2}\right)$ where $A$ is an invertible matrix). Any three points in the plane always lie on a translate of some lattice, so this recovers and extends the partial result (b) above. However, four arbitrary points need not be contained in a translate of a lattice. Indeed, the case $N=4$ of the conjecture is still open, and even the following special cases seem to be open.

Conjecture 9.2. If $g \in L^{2}(\mathbf{R})$ is nonzero, then each of the following is a linearly independent set of functions in $L^{2}(\mathbf{R})$ :

(a) $\left\{g(x), g(x-1), e^{2 \pi i x} g(x), e^{2 \pi i \sqrt{2} x} g(x-\sqrt{2})\right\}$,

(b) $\left\{g(x), g(x-1), e^{2 \pi i x} g(x), g(x-\pi)\right\}$.

Conjecture 9.2 remains open even if we impose the condition that $g$ be continuous (or smoother). Recently Rzeszotnik has settled a different specific four-point case, showing that $\left\{g(x), g(x-1), e^{2 \pi i x} g(x), g(x-\sqrt{2})\right\}$ is always linearly independent [106].

Finally, Kutyniok considered a generalized conjecture in [88], replacing the real line $\mathbf{R}$ by a locally compact Abelian group. Although the conjecture then becomes even more difficult to address, she was able to obtain some partial results. As we will mention later, even the seemingly trivial action of replacing $\mathbf{R}$ by $\mathbf{R}^{n}$ results in complications.

\subsection{Spanning, Independence, Frames, and Redundancy}

One motivation for the HRT Conjecture comes from looking at frames, which are possibly redundant or overcomplete collections of vectors in a Hilbert space which nonetheless provide basis-like representations of vectors in the 
space. Thus a frame "spans" the space in some sense, even though it may be "dependent." However, in infinite dimensions there are many shades of gray to the meanings of "spanning" and "independence." Some of the most important frames are "dependent" taken as a whole even though they have the property that every finite subset is linearly independent. One motivation for the HRT Conjecture is the question of whether the special class of Gabor frames (defined in Section 9.4) have this property.

In this section we provide some background on frames and the nuances of spanning and independence in infinite dimensions. For simplicity, the discussion will be kept to the setting of Banach spaces. Some of the definitions and discussion can be generalized easily to other settings, but other parts, especially the discussion of frames and their properties, are more explicitly Hilbert space theories, and there are subtleties in attempting to generalize those results beyond the Hilbert space setting.

We will state many facts but prove few of them; proofs and additional information can be found in the references provided in each section.

\subsubsection{Spanning and Independence in Finite Dimensions}

Spanning and independence are clear in finite dimensions. A set $\left\{f_{1}, \ldots, f_{M}\right\}$ of $M$ vectors in an $N$-dimensional vector space $H$ spans $H$ if for each vector $f \in H$ there exist scalars $c_{i}$ (not necessarily unique) such that $f=c_{1} f_{1}+$ $\cdots+c_{M} f_{M}$. This can only happen if $M \geq N$.

On the other hand, $\left\{f_{1}, \ldots, f_{M}\right\}$ is linearly independent if whenever a vector $f \in H$ can be written as $f=c_{1} f_{1}+\cdots+c_{M} f_{M}$, it can only be so written in one way, i.e., the scalars $c_{i}$ are unique if they exist at all. This can only happen if $M \leq N$.

When both of these happen simultaneously, we have a basis. In this case every $f \in H$ can be written as $f=c_{1} f_{1}+\cdots+c_{M} f_{M}$ for a unique choice of scalars $c_{i}$. This can only happen if $M=N$.

\subsubsection{Spanning in Infinite Dimensions}

For proofs, examples, and more information on bases, convergence of series, and related issues in normed spaces that are discussed in this section, we suggest the references [28], [64], [93], [98], [108], [114].

In a completely arbitrary vector space we can only define finite sums of vectors, because to define an infinite series we need a notion of convergence, and this requires a norm or metric or at least a topology. Thus, we define the finite linear span of a collection of vectors $\left\{f_{\alpha}\right\}_{\alpha \in J}$ to be

$$
\operatorname{span}\left(\left\{f_{\alpha}\right\}_{\alpha \in J}\right)=\left\{\sum_{i=1}^{N} c_{i} f_{\alpha_{i}}: N \in \mathbf{N}, c_{i} \in \mathbf{C}, \alpha_{i} \in J\right\} .
$$


We say that $\left\{f_{\alpha}\right\}_{\alpha \in J}$ spans $V$ if the finite span is all of $V$, i.e., every vector in $V$ equals some finite linear combination of the $f_{\alpha}$. We say that $\left\{f_{\alpha}\right\}_{\alpha \in J}$ is a Hamel basis if it both spans and is finitely linearly independent, or, equivalently, if every nonzero vector $f \in V$ can be written $f=\sum_{i=1}^{N} c_{i} f_{\alpha_{i}}$ for a unique choice of indices $\left\{\alpha_{i}\right\}_{i=1}^{N}$ and nonzero scalars $\left\{c_{i}\right\}_{i=1}^{N}$. For most vector spaces, Hamel bases are only known to exist because of the Axiom of Choice; in fact, the statement "Every vector space has a Hamel basis" is equivalent to the Axiom of Choice. Although Hamel bases are sometimes just called "bases," this is potentially confusing because if $V$ is a normed space, then the word basis is usually reserved for something different (see Definition 9.3).

As soon as we impose a little more structure on our vector space, we can often construct systems which are much more convenient than Hamel bases. For example, in a Banach space we have a norm, and hence can form "infinite linear combinations" by using infinite series. In particular, given a collection $\left\{f_{i}\right\}_{i \in \mathbf{N}}$ indexed by the natural numbers and given scalars $\left\{c_{i}\right\}_{i \in \mathbf{N}}$, we say the series $f=\sum_{i=1}^{\infty} c_{i} f_{i}$ converges and equals $f$ if $\left\|f-\sum_{i=1}^{N} c_{i} f_{i}\right\| \rightarrow 0$ as $N \rightarrow \infty$. Note that order in this series is important; if we change the order of indices we are not guaranteed that the series will still converge. If the convergence does not depend on the order it is called unconditional convergence, otherwise it is conditional convergence.

A related but distinct consequence of the fact that we have a norm is that we can form the closure of the finite linear span by constructing the set of all possible limits of finite linear combinations. This set is called the closed span, and is denoted $\overline{\operatorname{span}}\left(\left\{f_{i}\right\}_{i \in \mathbf{N}}\right)$. Given $f \in \overline{\operatorname{span}}\left(\left\{f_{i}\right\}_{i \in \mathbf{N}}\right)$, by definition there exist vectors $g_{N} \in \operatorname{span}\left(\left\{f_{i}\right\}_{i \in \mathbf{N}}\right)$ which converge to $f$. However, this is not the same as forming infinite linear combinations. While each $g_{N}$ is some finite linear combination of the $f_{i}$, it need not be true that we can write $g_{N}=\sum_{i=1}^{N} c_{i} f_{i}$ using a single sequence of scalars $\left\{c_{i}\right\}_{i \in \mathbf{N}}$.

Using these notions, we can form several variations on "spanning sets."

Definition 9.3. Let $\left\{f_{i}\right\}_{i \in \mathbf{N}}$ be a countable sequence of vectors in a Banach space $X$.

(a) $\left\{f_{i}\right\}_{i \in \mathbf{N}}$ is complete (or total or fundamental) if $\overline{\operatorname{span}}\left(\left\{f_{i}\right\}_{i \in \mathbf{N}}\right)=X$, i.e., for each $f \in X$ and each $N \in \mathbf{N}$ there exist scalars $\left\{c_{N, i}(f)\right\}_{i \in \mathbf{N}}$ such that $\sum_{i=1}^{N} c_{N, i}(f) f_{i} \rightarrow f$ as $N \rightarrow \infty$.

(b) $\left\{f_{i}\right\}_{i \in \mathbf{N}}$ has Property $\mathrm{S}$ if for each $f \in X$ there exist scalars $\left\{c_{i}(f)\right\}_{i \in \mathbf{N}}$ such that

$$
f=\sum_{i=1}^{\infty} c_{i}(f) f_{i}
$$

(c) $\left\{f_{i}\right\}_{i \in \mathbf{N}}$ is a quasibasis if it has Property $S$ and for each $i \in \mathbf{N}$ the mapping $f \mapsto c_{i}(f)$ is linear and continuous (and hence defines an element of the dual space $\left.X^{*}\right)$. 
(d) $\left\{f_{i}\right\}_{i \in \mathbf{N}}$ is a basis or Schauder basis if it has Property $S$ and for each $f \in X$ the scalars $\left\{c_{i}(f)\right\}_{i \in \mathbf{N}}$ are unique.

Completeness is a weak property. The definition says that there are finite linear combinations of the $f_{i}$ that converge to $f$, but the scalars needed can change completely as the length $N$ of the linear combination increases. On the other hand, unlike the other properties there exists a nice, simple characterization of complete sequences. For the case of a Hilbert space it is:

$$
\left\{f_{i}\right\}_{i \in \mathbf{N}} \text { is complete } \Longleftrightarrow \text { only } f=0 \text { is orthogonal to every } f_{i}
$$

(for a general Banach space we just have to take $f$ to lie in the dual space $X^{*}$ ). Consequently, if $\left\{f_{i}\right\}_{i \in \mathbf{N}}$ is complete, then every $f \in H$ is uniquely determined by the sequence of inner products $\left\{\left\langle f, f_{i}\right\rangle\right\}_{i \in \mathbf{N}}$, or in other words, the analysis operator $T(f)=\left\{\left\langle f, f_{i}\right\rangle\right\}_{i \in \mathbf{N}}$ is an injective mapping into the space of all sequences. However, this doesn't give us an algorithm for constructing $f$ from those inner products, and in general there need not exist a stable way to do so, i.e., $T^{-1}$ need not be continuous.

Property S seems to have no standard name in the literature (hence the uncreative name invented here), perhaps because it is not really a very useful concept by itself. In particular, the definition fails to provide us with a stable algorithm for finding a choice of coefficients $c_{i}(f)$ that can be used to represent $f$. The definition of quasibasis addresses this somewhat by requiring that each mapping $f \mapsto c_{i}(f)$ be continuous (for more details on quasibases, see [86] and the references therein). However, this is still not sufficient in most applications, as it is not so much the continuity of each individual map $f \mapsto c_{i}(f)$ that is important but rather the continuity of the mapping from $f$ to the entire associated sequence $\left\{c_{i}(f)\right\}_{i \in \mathbf{N}}$. In other words, in concrete applications there is often some particular associated Banach space $X_{d}$ of sequences (imposed by the context), and the mapping $f \mapsto\left\{c_{i}(f)\right\}_{i \in \mathbf{N}}$ must be a continuous linear map of $X$ into $X_{d}$. Specializing to the Hilbert space case, this is one of the ideas behind the definition of frames (see Section 9.3.3).

Imposing uniqueness seems to be a natural requirement, and in fact, it can be shown that even though the definition of basis does not include the requirement that $f \mapsto c_{i}(f)$ be continuous, this follows automatically from the uniqueness assumption (and the fact that we are using norm convergence). Thus every basis is actually a quasibasis. Unfortunately, in many contexts uniqueness is simply too restrictive. For example, this is the case for Gabor systems (compare the Balian-Low Theorem, Theorem 9.16 below). The terms "basis" and "Schauder basis" are used interchangeably in the Banach space setting.

We can summarize the relations among the "spanning type" properties introduced so far by the following implications:

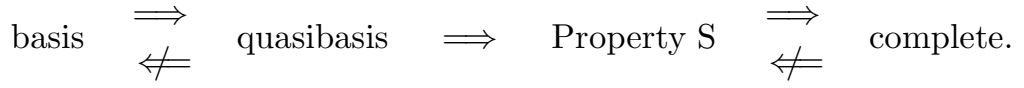


It seems unclear whether every system with Property S must actually be a quasibasis (compare [54]), but the other implications are known to not be reversible in general (even in a Hilbert space).

\subsubsection{Frames}

In the Hilbert space setting, frames are a class of sequences which not only are quasibases but also provide stable reconstruction formulas. Frames were first introduced by Duffin and Schaeffer [46], and that paper still provides instructive reading today. For proofs and more information on frames and the results presented in this section, we suggest [22], [28], [41], [55], [64], [71], [114]. For an interesting recent paper that deals with the issue of extending frames beyond the Hilbert space setting, see [58].

Definition 9.4. A sequence $\mathcal{F}=\left\{f_{i}\right\}_{i \in \mathbf{N}}$ is a frame for a Hilbert space $H$ if there exist constants $A, B>0$, called frame bounds, such that

$$
\forall f \in H, \quad A\|f\|^{2} \leq \sum_{i=1}^{\infty}\left|\left\langle f, f_{i}\right\rangle\right|^{2} \leq B\|f\|^{2} .
$$

The largest possible value for $A$ and the smallest possible value for $B$ are the optimal frame bounds. If we can take $A=B$ in (9.2), then we say the frame is tight.

Every orthonormal basis is a tight frame, because an orthonormal basis satisfies the Parseval/Plancherel formula, which is exactly (9.2) for the case $A=B=1$. However, not every frame is an orthonormal basis, even if $A=$ $B=1$. For example, if $\left\{e_{i}\right\}_{i \in \mathbf{N}}$ and $\left\{f_{i}\right\}_{i \in \mathbf{N}}$ are both orthonormal bases for $H$, then $\left\{\frac{1}{\sqrt{2}} e_{i}\right\}_{i \in \mathbf{N}} \cup\left\{\frac{1}{\sqrt{2}} f_{i}\right\}_{i \in \mathbf{N}}$ is a frame with $A=B=1$ that is not an orthonormal basis. A frame for which we can take $A=B=1$ is often called a Parseval frame or a normalized tight frame (but the latter term is confusing because some papers, such as [16], use it differently).

A frame need not be a basis (if not, it is said to be redundant or overcomplete). However, every frame is a quasibasis. A sketch of why this is true starts with the analysis operator $T: H \rightarrow \ell^{2}$ given by $T(f)=\left\{\left\langle f, f_{i}\right\rangle\right\}_{i \in \mathbf{N}}$ and the frame operator $S: H \rightarrow H$ given by $S f=T^{*} T f=\sum_{i}\left\langle f, f_{i}\right\rangle f_{i}$. The frame definition implies that $T$ is a bounded injective mapping of $H$ onto a closed subset of $\ell^{2}$, and the inverse map $T^{-1}: \operatorname{range}(T) \rightarrow H$ is also bounded. Further, it can be shown that the series defining $S f$ converges for every $f$, and that $S$ is actually a positive definite, invertible mapping of $H$ onto itself. Writing out and rearranging the equalities $f=S S^{-1} f=S^{-1} S f$ then gives the frame expansions

$$
\forall f \in H, \quad f=\sum_{i=1}^{\infty}\left\langle f, \tilde{f}_{i}\right\rangle f_{i}=\sum_{i=1}^{\infty}\left\langle f, f_{i}\right\rangle \tilde{f}_{i},
$$


where $\tilde{f}_{i}=S^{-1} f_{i}$. Thus both the frame $\left\{f_{i}\right\}_{i \in \mathbf{N}}$ and its canonical dual frame $\left\{\tilde{f}_{i}\right\}_{i \in \mathbf{N}}$ are quasibases. We even have simple (and computable) formulas for the coefficients, namely, $c_{i}(f)=\left\langle f, \tilde{f}_{i}\right\rangle$ for the frame and $c_{i}(f)=\left\langle f, f_{i}\right\rangle$ for the dual frame. In general, however, these scalars need not be unique. Trivial examples of nonuniqueness are a frame which includes some zero vectors as elements, or the union of two orthonormal bases. For a nontrivial example, see Example 9.5.

Note that if we rearrange the elements of the frame then we still have a frame, because the series in (9.2) is a series of nonnegative numbers, and hence if it converges then any rearrangement also converges. It follows that the frame expansions in (9.3) converge unconditionally. This is one of many stability properties enjoyed by frames. As a consequence any countable index set can be used to specify the elements of a frame.

Although the scalars in the frame expansions in (9.3) need not be unique, out of all the possible sequences $\left\{c_{i}\right\}_{i \in \mathbf{N}}$ such that $f=\sum_{i} c_{i} f_{i}$, the frame coefficients have minimal energy, i.e., $\sum_{i}\left|\left\langle f, \tilde{f}_{i}\right\rangle\right|^{2} \leq \sum_{i}\left|c_{i}\right|^{2}$, and equality holds only when $\left|c_{i}\right|=\left|\left\langle f, \tilde{f}_{i}\right\rangle\right|$ for all $i$. This does not imply that $\sum_{i}\left|c_{i}\right|^{2}$ is finite; compare [31], [63], [76]. In particular applications, we can make use of the fact that different choices of coefficients can be used to search for other noncanonical dual functions $\tilde{f}$ (possibly even from a larger space) that still provide frame expansions but may possess extra properties important for the application at hand. Some papers on noncanonical duals or on minimizing with respect to other criteria than energy are [27], [30], [92].

In finite dimensions, frames are easy to characterize:

- A collection $\left\{f_{1}, \ldots, f_{M}\right\}$ is a frame for a finite-dimensional Hilbert space $H$ if and only if $\left\{f_{1}, \ldots, f_{M}\right\}$ spans $H$. Thus, in a finite-dimensional space, a collection is a basis if and only if it is a linearly independent frame.

In particular, every finite set of vectors $\left\{f_{1}, \ldots, f_{M}\right\}$ in a Hilbert space is a frame for the subspace $S=\operatorname{span}\left(\left\{f_{1}, \ldots, f_{M}\right\}\right)$.

Finite-dimensional frames have many important applications, and there remain many deep and difficult mathematical questions concerning them, such as characterizing frames which have certain useful properties. We suggest [16], [24], [47], [109] as some interesting papers on "finite frames;" in particular, the last paper discusses links between finite frames and other areas such as discrete geometry and sphere packings.

The following illustrative example shows that the relationship between frames and linear independence is more complicated in infinite dimensions.

Example 9.5. Let $e_{n}(x)=e^{2 \pi i n x}$. The system of exponentials $\left\{e_{n}\right\}_{n \in \mathbf{Z}}$ is an orthonormal basis for the Hilbert space $L^{2}[0,1]$. What happens if we change the frequencies from integers $n$ to integer multiples $n \beta$ of some $\beta>0$ ? That is, setting $e_{n \beta}(x)=e^{2 \pi i n \beta x}$ but keeping the domain $[0,1]$, we ask what properties the new sequence $\left\{e_{n \beta}\right\}_{n \in \mathbf{Z}}$ has in $L^{2}[0,1]$. 
Case $\beta>1$. Note that each $e_{n \beta}$ is $\frac{1}{\beta}$-periodic and $\frac{1}{\beta}<1$. Every finite linear combination will likewise be $\frac{1}{\beta}$-periodic, as will any element of the closed span. Hence the closed span cannot be all of $L^{2}[0,1]$, since there are many elements of $L^{2}[0,1]$ that are not $\frac{1}{\beta}$-periodic, such as $f(x)=x$. Also, the vector

$$
f(x)=\left\{\begin{aligned}
1, & 0 \leq x<\varepsilon \\
-1, & \frac{1}{\beta} \leq x<\frac{1}{\beta}+\varepsilon \\
0, & \text { otherwise }
\end{aligned}\right.
$$

is orthogonal to every $e_{n \beta}$. Thus $\left\{e_{n \beta}\right\}_{n \in \mathbf{Z}}$ is incomplete when $\beta>1$.

Case $\beta<1$. Suppose $\beta<1$. Since $\left\{\sqrt{\beta} e^{2 \pi i n \beta x}\right\}_{n \in \mathbf{Z}}$ is an orthonormal basis for the space $L^{2}\left[0, \frac{1}{\beta}\right]$, we have

$$
\forall f \in L^{2}\left[0, \frac{1}{\beta}\right], \quad \sum_{n \in \mathbf{Z}}\left|\left\langle f, e_{n \beta}\right\rangle\right|^{2}=\frac{1}{\beta}\|f\|_{2}^{2} .
$$

Given $f \in L^{2}[0,1]$, extend it to $\left[0, \frac{1}{\beta}\right]$ by setting $f(x)=0$ for $1<x \leq \frac{1}{\beta}$. Then we can apply (9.4), but because of the zero extension, the norm and inner product are from $L^{2}[0,1]$. In other words, (9.4) holds for $f \in L^{2}[0,1]$, so $\left\{e_{n \beta}\right\}_{n \in \mathbf{Z}}$ is a tight frame for $L^{2}[0,1]$ with frame bounds $A=B=\frac{1}{\beta}$. In fact, this set is the image of an orthonormal basis for $L^{2}\left[0, \frac{1}{\beta}\right]$ under the orthogonal projection $f \mapsto f \cdot \chi_{[0,1]}$. This can be generalized; from an operator theory viewpoint frames can be viewed in terms of projections and vice versa [61].

In any case, $\left\{e_{n \beta}\right\}_{n \in \mathbf{Z}}$ is a tight frame when $\beta<1$, with frame bounds $A=B=\frac{1}{\beta}$ and frame operator $S=A I=\frac{1}{\beta} I$. Hence the dual frame elements are $\tilde{e}_{n \beta}=S^{-1}\left(e_{n \beta}\right)=\beta e_{n \beta}$, and frame expansions are trivial to compute. As a consequence, we can see directly that this frame is not a basis, because the coefficients in the frame expansion are not unique. For example, the constant function $e_{0}(x)=1$ has two expansions:

$$
\sum_{n \in \mathbf{Z}} \delta_{n} e_{n \beta}=e_{0}=\sum_{n \in \mathbf{Z}}\left\langle e_{0}, \tilde{e}_{n \beta}\right\rangle e_{n \beta}
$$

where $\delta_{n}=1$ when $n=0$ and 0 otherwise. Since $\left\langle e_{0}, \tilde{e}_{n \beta}\right\rangle=\frac{1-e^{2 \pi i n \beta}}{2 \pi i n}$ for $n \neq 0$, the two expansions in (9.5) are in fact different.

By rearranging (9.5), we can write

$$
e_{0}=\sum_{n \neq 0} c_{n} e_{n \beta}
$$

for appropriate scalars $c_{n}$, i.e., $e_{0}$ is expressible in terms of the other frame elements (this cannot happen in a basis). It can actually be shown that the proper subset $\left\{e_{n \beta}\right\}_{n \neq 0}$ of the original frame $\left\{e_{n \beta}\right\}_{n \in \mathbf{Z}}$ is still a frame for 
$L^{2}[0,1]$, although it is no longer tight. Thus the original frame is redundant in the sense that a proper subset is still a frame. For this particular frame it can even be shown that there is an infinite set $J \subset \mathbf{Z}$ such that $\left\{e_{n \beta}\right\}_{n \in \mathbf{Z} \backslash J}$ is still a frame for $L^{2}[0,1]$, so in some sense the original frame is extremely redundant. Yet it is linearly independent using the standard abstract linear algebra definition of independence, namely, every finite subset of $\left\{e_{n \beta}\right\}_{n \in \mathbf{Z}}$ is linearly independent! For, if we write a finite linear combination as $\sum_{n=-N}^{N} c_{n} e^{2 \pi i n \beta x}=\sum_{n=-N}^{N} c_{n} z^{n}$ where $z=e^{2 \pi i \beta x}$, then we can apply the Fundamental Theorem of Algebra to conclude that such a polynomial cannot vanish for all $x$ unless it is the trivial polynomial. Thus $\left\{e_{n \beta}\right\}_{n \in \mathbf{Z}}$ is very redundant taken as a whole even though every finite subset is independent.

Remark 9.6. a. The value $\frac{1}{\beta}$ is sometimes called the density of the system $\left\{e_{n \beta}\right\}_{n \in \mathbf{Z}}$. The value $\frac{1}{\beta}=1$ is the Nyquist density for the exponentials; at the Nyquist density the system is an orthonormal basis, at lower densities $\left(\frac{1}{\beta}<1\right)$ it is incomplete and at higher densities $\left(\frac{1}{\beta}>1\right)$ it is an overcomplete frame. The Classical (or Shannon) Sampling Theorem for bandlimited signals is an immediate consequence of the frame properties of the exponentials; for more details on the Sampling Theorem we refer to Benedetto's text [13].

b. Many of the statements made about the system $\left\{e_{n \beta}\right\}_{n \in \mathbf{Z}}$ have analogues for "irregular" sequences of exponentials of the form $\left\{e_{\lambda_{n}}\right\}_{n \in \mathbf{Z}}=$ $\left\{e^{2 \pi i \lambda_{n} x}\right\}_{n \in \mathbf{Z}}$, where the $\lambda_{n}$ are arbitrary points in $\mathbf{R}$. In these statements the Beurling density of the set $\left\{\lambda_{n}\right\}_{n \in \mathbf{Z}}$ replaces the value $\frac{1}{\beta}$. We suggest [114] and [60] as starting points for more details on this topic. In Section 9.4.1 below, we present some analogous results for irregular Gabor systems, and Beurling density is defined precisely there.

c. Frames of exponentials are very special types of frames, and only some of the statements made in Example 9.5 carry over to general frames. For an arbitrary frame, it can be shown that if $\left\{f_{i}\right\}_{i \in \mathbf{N}}$ is a redundant frame, then there exists at least a finite set $F$ such that $\left\{f_{i}\right\}_{i \notin F}$ is still a frame. However, in general it need not be the case that infinitely many elements can be removed yet leave a frame [6], nor that there need be some subset of the frame that is a basis [29], [107]. The Feichtinger Conjecture is the statement that every frame $\mathcal{F}=\left\{f_{i}\right\}_{i \in I}$ satisfying $\inf \left\|f_{i}\right\|>0$ can be written as a finite union of subsequences that are Riesz bases for their closed spans. This conjecture is open and has recently been shown to be equivalent to the deep and longstanding Kadison-Singer Conjecture in operator theory, which has been open since 1959 [85]; see [26], [23] for the proof of the equivalence.

\subsubsection{Independence in Infinite Dimensions}

We explore independence in more detail in this section. For proofs and more information, see [28], [64], [93], [98], [108], [114]. 
The following are several shades of gray in the possible definition of independence.

Definition 9.7. Let $\left\{f_{i}\right\}_{i \in \mathbf{N}}$ be a countable sequence of elements in a Banach space $X$.

(a) $\left\{f_{i}\right\}_{i \in \mathbf{N}}$ is a basis or Schauder basis if for each $f \in X$ there exist unique scalars $c_{i}$ such that $f=\sum_{i} c_{i} f_{i}$.

(b) $\left\{f_{i}\right\}_{i \in \mathbf{N}}$ is minimal if for each $j \in \mathbf{N}$, the vector $f_{j}$ does not lie in $\overline{\operatorname{span}}\left(\left\{f_{i}\right\}_{i \neq j}\right)$. Equivalently (via Hahn-Banach), there must exist a sequence $\left\{\tilde{f}_{i}\right\}_{i \in \mathbf{N}}$ in the dual space $X^{*}$ that is biorthogonal to $\left\{f_{i}\right\}_{i \in \mathbf{N}}$, i.e., $\left\langle f_{i}, \tilde{f}_{j}\right\rangle=1$ if $i=j$ and 0 if $i \neq j$.

(c) $\left\{f_{i}\right\}_{i \in \mathbf{N}}$ is $\omega$-independent if the series $\sum_{i=1}^{\infty} c_{i} f_{i}$ can converge and equal the zero vector only when every $c_{i}=0$.

(d) $\left\{f_{i}\right\}_{i \in \mathbf{N}}$ is finitely independent (or simply independent) if every finite subset is independent, i.e., for any $N$ we have $\sum_{i=1}^{N} c_{i} f_{i}=0$ if and only if $c_{1}=\cdots=c_{N}=0$.

For example, consider the system of exponentials $\left\{e_{n \beta}\right\}_{n \in \mathbf{Z}}$ described in Example 9.5. We have already seen that the system is a basis only for $\beta=1$. If $\beta>1$ then it is not even complete, while if $\beta<1$ then it is not a basis because we showed explicitly in (9.5) that the vector $e_{0}$ has two different series representations. Additionally, equation (9.6) implies that $e_{0}$ lies in the closure of $\operatorname{span}\left(\left\{e_{n \beta}\right\}_{n \neq 0}\right)$, so the system is not minimal. Further, by subtracting $e_{0}$ from both sides of (9.6) we obtain a nontrivial infinite series that converges and equals the zero vector, so the system is not $\omega$-independent. Even so, that system is finitely independent.

The following implications among these properties hold, none of which is reversible in general (even in a Hilbert space):

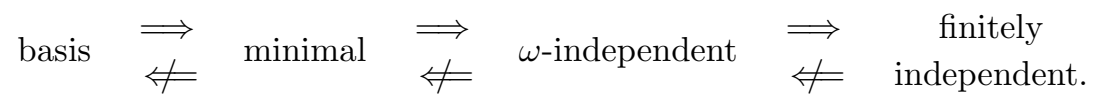

One technical point is that the definition of basis really combines aspects of both spanning and independence, i.e., a basis is necessarily complete and has Property S. Adding completeness doesn't change the implications above, e.g., every basis is both minimal and complete, but a minimal sequence that is complete need not be a basis (a sequence which is both minimal and complete is sometimes called an exact sequence). On the other hand, Property $\mathrm{S}$ is exactly what is missing for a minimal or $\omega$-independent sequence to be a basis, for with either of those hypotheses, once we know that an infinite series $\sum_{i=1}^{\infty} c_{i} f_{i}$ converges, we can conclude that the coefficients are unique. However, as shown by the example of the exponentials, finite independence combined with Property S is not sufficient to ensure that we have a basis. Thus we have the following equivalences: 


$$
\text { basis } \Longleftrightarrow \begin{gathered}
\text { minimal with } \\
\text { Property } \mathrm{S}
\end{gathered} \Longleftrightarrow \begin{gathered}
\text { w-independent } \\
\text { with Property S, }
\end{gathered}
$$

and each of these implies finite independence, but not conversely: a finitely independent sequence which has Property S need not be a basis. Similarly, combining the various independence criteria with a frame hypothesis, we obtain the following result, which should be compared to Example 9.5, where we showed that a frame which is finitely independent need not be a basis.

Theorem 9.8. Let $\left\{f_{i}\right\}_{i \in \mathbf{N}}$ be a countable sequence of elements in a Hilbert space $H$. Then the following statements are equivalent.

(a) $\left\{f_{i}\right\}_{i \in \mathbf{N}}$ is a frame and a basis for $H$.

(b) $\left\{f_{i}\right\}_{i \in \mathbf{N}}$ is a frame and a minimal sequence.

(c) $\left\{f_{i}\right\}_{i \in \mathbf{N}}$ is a frame and an $\omega$-independent sequence.

(d) $\left\{f_{i}\right\}_{i \in \mathbf{N}}$ is a Riesz basis for $H$, i.e., it is the image of an orthonormal basis for $H$ under a continuous linear bijection.

(e) $\left\{f_{i}\right\}_{i \in \mathbf{N}}$ is a bounded unconditional basis for $H$, i.e., for each $f \in H$ there are unique scalars $c_{i}$ such that $f=\sum_{i} c_{i} f_{i}$, where the series converges unconditionally, and additionally $0<\inf _{i}\left\|f_{i}\right\| \leq \sup _{i}\left\|f_{i}\right\|<\infty$.

The most common terms used to describe a frame which satisfies the equivalent conditions of Theorem 9.8 are Riesz basis and exact frame. Continuous linear bijections are known by a variety of names, including topological isomorphisms, continuously invertible maps, or even just invertible maps.

One set of extra hypotheses needed for a finitely independent set to be a Riesz basis is given in the following theorem quoted from [28, Prop. 6.1.2] and originally proved in [33], [87].

Theorem 9.9. Let $\left\{f_{i}\right\}_{i \in \mathbf{N}}$ be a countable sequence of elements in a Hilbert space $H$, and let $A_{N}$ be the optimal lower frame bound for $\left\{f_{i}\right\}_{i=1}^{N}$ as a frame for its span. Then the following statements are equivalent.

(a) $\left\{f_{i}\right\}_{i \in \mathbf{N}}$ is a Riesz basis for $H$.

(b) $\left\{f_{i}\right\}_{i \in \mathbf{N}}$ is finitely independent and $\inf A_{N}>0$.

(c) $\left\{f_{i}\right\}_{i \in \mathbf{N}}$ is finitely independent and $\lim _{N \rightarrow \infty} A_{N}$ exists and is positive.

If $\left\{e_{n}\right\}_{n \in \mathbf{N}}$ is an orthonormal basis for $H$, then the renormalized sequence $\left\{\frac{e_{n}}{n}\right\}_{n \in \mathbf{N}}$ is a trivial example of a Schauder basis that is not a Riesz basis. On the other hand, here is a nontrivial example where all the elements have the same norm.

Example 9.10. Fix $0<\alpha<\frac{1}{2}$; then $|x|^{\alpha}$ and $|x|^{-\alpha}$ both belong to $L^{2}\left[-\frac{1}{2}, \frac{1}{2}\right]$. Hence $\left\{e^{2 \pi i n x}|x|^{\alpha}\right\}_{n \in \mathbf{Z}}$ and $\left\{e^{2 \pi i n x}|x|^{-\alpha}\right\}_{n \in \mathbf{Z}}$ are biorthogonal systems in $L^{2}\left[-\frac{1}{2}, \frac{1}{2}\right]$ and therefore are minimal. It is a much more difficult result, due to 
Babenko [3], that these systems are actually Schauder bases for $L^{2}\left[-\frac{1}{2}, \frac{1}{2}\right]$ (see also the discussion in [108, pp. 351-354]). Since these systems are obtained by taking the orthonormal basis $\left\{e^{2 \pi i n x}\right\}_{n \in \mathbf{Z}}$ and performing an operation that is not a continuous bijection (i.e., multiplying by the function $|x|^{\alpha}$ which has a zero or by the unbounded function $|x|^{-\alpha}$ ), they are not Riesz bases. On the other hand, these systems do possess one but not both frame bounds. Specifically, $\left\{e^{2 \pi i n x}|x|^{\alpha}\right\}_{n \in \mathbf{Z}}$ is a Bessel sequence while $\left\{e^{2 \pi i n x}|x|^{-\alpha}\right\}_{n \in \mathbf{Z}}$ possesses a lower frame bound.

\subsection{Gabor Frames}

Now we will discuss the special class of frames known as Gabor frames (they are also often called Weyl-Heisenberg frames). This is one part of the broader field of time-frequency analysis. We will survey some results on Gabor frames, with the choice of topics inspired by issues relating to independence and the HRT Conjecture. As a result, many important topics and contributions by many founders of the field are not included. For a more complete introduction to time-frequency analysis we recommend Gröchenig's text [55], and for surveys and basic information on Gabor frames we suggest [22], [28], [41], [71], [81].

\subsubsection{Density and Gabor Frames}

In this section we give some background on Gabor systems and Gabor frames.

Definition 9.11. Let $g \in L^{2}(\mathbf{R})$. The translation of $g$ by $a \in \mathbf{R}$ is $T_{a} g(x)=$ $g(x-a)$, and the modulation of $g$ by $b \in \mathbf{R}$ is $M_{b} g(x)=e^{2 \pi i b x} g(x)$. The compositions

$$
M_{b} T_{a} g(x)=e^{2 \pi i b x} g(x-a)
$$

and

$$
T_{a} M_{b}(x)=e^{2 \pi i b(x-a)} g(x-a)=e^{-2 \pi i a b} M_{b} T_{a} g(x)
$$

are time-frequency shifts of $g$. If $\Lambda \subset \mathbf{R}^{2}$ then the Gabor system generated by $g$ and $\Lambda$ is

$$
\mathcal{G}(g, \Lambda)=\left\{M_{b} T_{a} g\right\}_{(a, b) \in \Lambda} .
$$

If a Gabor system is a frame for $L^{2}(\mathbf{R})$, then it is called a Gabor frame.

Note that $\Lambda$ should technically be regarded as a sequence of points in $\mathbf{R}^{2}$ rather than a subset, because frames can allow duplicate elements, and so we should allow repetitions of points in $\Lambda$. However, for simplicity we usually just write $\Lambda \subset \mathbf{R}^{2}$ even though we mean that $\Lambda$ is a sequence. Typically, we are interested in countable sets $\Lambda$, and the most common case is where $\Lambda$ is the rectangular lattice $\Lambda=\alpha \mathbf{Z} \times \beta \mathbf{Z}$. The cases of general lattices $\Lambda=A\left(\mathbf{Z}^{2}\right)$ and nonlattice or "irregular" sets of time-frequency shifts $\Lambda$ are also very interesting and important. 
Example 9.12. (a) $\mathcal{G}\left(\chi_{[0,1)}, \mathbf{Z}^{2}\right)$ is a Gabor orthonormal basis for $L^{2}(\mathbf{R})$.

(b) Gabor studied the system $\mathcal{G}\left(e^{-x^{2}}, \mathbf{Z}^{2}\right)$ in the context of information theory [51]. It can be shown via Zak transform techniques that this system is not a frame, and is overcomplete by exactly one element [102], [77]. That is, one element may be removed and leave a complete set, but not two elements. Thus $\mathcal{G}\left(e^{-x^{2}}, \mathbf{Z}^{2} \backslash(0,0)\right)$ is minimal and complete. However, it is not a basis for $L^{2}(\mathbf{R})[49$, p. 168]. The Zak transform is briefly discussed in Section 9.5.3.

(c) It is easy to create specific Gabor frames. For example, if $g \in L^{2}(\mathbf{R})$ is supported in $\left[0, \frac{1}{\beta}\right]$ and satisfies $\sum_{n \in \mathbf{Z}}|g(x-n \alpha)|^{2}=1$, then $\mathcal{G}(g, \alpha \mathbf{Z} \times \beta \mathbf{Z})$ is a tight frame. Note that this requires $\alpha \beta \leq 1$; compare Theorem 9.13 below. If $\alpha \beta=1$, then $g$ will be discontinuous; compare Theorem 9.16 below. However, if $\alpha \beta<1$, then we can create frames where $g$ is as smooth as we like, even infinitely differentiable. These frames, and their wavelet analogues, are the "painless nonorthogonal expansions" of [42]. For recent higher-dimensional "irregular" frame constructions in a similar spirit, see [1].

Let us mention that Gabor systems have a natural connection to representation theory. The time-frequency plane $\mathbf{R}^{2}$ appearing in the definition of a Gabor system is really the Heisenberg group in disguise. One form of the Heisenberg group is $\mathbf{H}=\mathbf{T} \times \mathbf{R} \times \mathbf{R}$, where $\mathbf{T}=\{z \in \mathbf{C}:|z|=1\}$, with a group operation that is induced by considering a point $(z, a, b) \in \mathbf{H}$ to correspond to the operator $z M_{b} T_{a}$ defined by $\left(z M_{b} T_{a} f\right)(x)=z e^{2 \pi i b x} f(x-a)$. That is, $(z, a, b) \mapsto z M_{b} T_{a}$ is required to be an injective homomorphism of $\mathbf{H}$ into the set of unitary mappings of $L^{2}(\mathbf{R})$ onto itself. Since

$$
\left(z M_{b} T_{a}\right) \circ\left(w M_{d} T_{c}\right)=e^{-2 \pi i a d} z w M_{b+d} T_{a+c},
$$

the group operation on $\mathbf{H}$ is therefore

$$
(z, a, b) \cdot(w, c, d)=\left(e^{-2 \pi i a d} z w, a+c, b+d\right) .
$$

This makes $\mathbf{H}$ a non-Abelian group, even though as a set it is simply the Cartesian product $\mathbf{T} \times \mathbf{R} \times \mathbf{R}$. To be a little more precise, the Heisenberg group is usually defined by considering compositions of "symmetric" time-frequency shifts $M_{\frac{b}{2}} T_{a} M_{\frac{b}{2}}$, and so the standard definitions differ in normalization from what is given here. For precise details we refer to [55, Ch. 9], [49]. The mapping $(z, a, b) \mapsto z M_{b} T_{a}$ is (except for normalization) the Schrödinger representation of $\mathbf{H}$. Although the unit modulus scalars $z$ are needed to define the group operation in $\mathbf{H}$, they play no role in many parts of time-frequency analysis, and hence we often end up dealing with the time-frequency plane $\mathbf{R}^{2}$ rather than $\mathbf{H}$.

Gabor systems defined with respect to rectangular lattices are especially nice, and have connections to the theory of $C^{*}$ and von Neumann algebras (for 
more on this connection see [44], [52], [61]). Such rectangular Gabor systems have properties that are very reminiscent of the system of exponentials discussed in Example 9.5. Specifically, the following result holds; note the similar role that the value $\alpha \beta$ plays as compared to the value of $\beta$ in Example 9.5.

Theorem 9.13. Let $g \in L^{2}(\mathbf{R})$ and let $\Lambda=\alpha \mathbf{Z} \times \beta \mathbf{Z}$ where $\alpha, \beta>0$. Then the Gabor system $\mathcal{G}(g, \alpha \mathbf{Z} \times \beta \mathbf{Z})=\left\{e^{2 \pi i \beta n x} g(x-\alpha k)\right\}_{k, n \in \mathbf{Z}}$ satisfies the following.

(a) If $\mathcal{G}(g, \alpha \mathbf{Z} \times \beta \mathbf{Z})$ is a frame for $L^{2}(\mathbf{R})$, then $0<\alpha \beta \leq 1$.

(b) If $\mathcal{G}(g, \alpha \mathbf{Z} \times \beta \mathbf{Z})$ is a frame for $L^{2}(\mathbf{R})$, then it is a Riesz basis if and only if $\alpha \beta=1$.

(c) If $\alpha \beta>1$, then $\mathcal{G}(g, \alpha \mathbf{Z} \times \beta \mathbf{Z})$ is incomplete in $L^{2}(\mathbf{R})$.

Theorem 9.13 has a long history that we cannot do justice to here. We mention only the following facts, and for more detailed history and references refer to the expositions in [17], [41], [55], [81].

Part (c) of Theorem 9.13 was proved the case that $\alpha \beta$ is rational by Daubechies [40] and for arbitrary $\alpha \beta$ by Baggett [4]. Daubechies' proof relied on the Zak transform, while Baggett used the theory of von Neumann algebras. Daubechies also noted that a proof for general $\alpha \beta$ can be inferred from results of Rieffel [104] on $C^{*}$ algebras. Another proof of part (c) based on von Neumann algebras is given in [44], and a new proof appears in [20].

Since every frame is complete, part (a) is of course a consequence of part (c), but we state it separately to emphasize the contrast with the case of irregular Gabor systems as stated in Theorem 9.14 below. A simple proof of part (a) was given by Janssen [79]. This proof relies on the algebraic structure of the rectangular lattice $\alpha \mathbf{Z} \times \beta \mathbf{Z}$ and the remarkable Wexler-Raz Theorem for Gabor frames $\mathcal{G}(g, \alpha \mathbf{Z} \times \beta \mathbf{Z})$. For more on Wexler-Raz see [79], the expositions in [55, Sec. 7.5], [81], [62], and the rigorous proofs in [80], [44].

In part (b), given a Gabor frame $\mathcal{G}(g, \alpha \mathbb{Z} \times \beta \mathbb{Z})$, it is easy to prove using the Zak transform that if $\alpha \beta=1$, then this frame must must be a Riesz basis. However, the converse is not as easy, and was first proved by Ramanathan and Steger in [103] as a special case of a much more general result (Theorem 9.14) discussed below. Today there are "straightforward" proofs of part (b), again based on Wexler-Raz.

In Theorem 9.13, the value $\alpha \beta$ that distinguishes between the various cases is a measurement of the "size" of the lattice $\alpha \mathbf{Z} \times \beta \mathbf{Z}$, as it is the area of a fundamental domain for that lattice. In the irregular setting there is no analogue of fundamental domain, and instead it is the Beurling density of $\Lambda$ that distinguishes between the various cases. Beurling density measures in some sense the average number of points inside unit squares. Because the points are not uniformly distributed, there is not a single definition, but rather we obtain lower and upper limits to the average density. More precisely, we count the average number of points inside squares of larger and larger radii and take the limit, yielding the definitions 


$$
\begin{aligned}
& D^{-}(\Lambda)=\liminf _{r \rightarrow \infty} \inf _{z \in \mathbf{R}^{2}} \frac{\#\left(\Lambda \cap Q_{r}(z)\right)}{r^{2}}, \\
& D^{+}(\Lambda)=\limsup _{r \rightarrow \infty} \sup _{z \in \mathbf{R}^{2}} \frac{\#\left(\Lambda \cap Q_{r}(z)\right)}{r^{2}},
\end{aligned}
$$

for the lower and upper Beurling densities of $\Lambda$. Here $Q_{r}(z)$ is the square in $\mathbf{R}^{2}$ centered at $z$ with side lengths $r$ and \# $E$ denotes cardinality. Using this notation, we can give necessary conditions for a Gabor system to be a frame or Riesz basis for $L^{2}(\mathbf{R})$, as follows.

Theorem 9.14. Let $g \in L^{2}(\mathbf{R})$ and let $\Lambda \subset \mathbf{R}^{2}$ be given. Then the Gabor system $\mathcal{G}(g, \Lambda)$ has the following properties.

(a) If $\mathcal{G}(g, \Lambda)$ is a frame for $L^{2}(\mathbf{R})$, then $1 \leq D^{-}(\Lambda) \leq D^{+}(\Lambda)<\infty$.

(b) If $\mathcal{G}(g, \Lambda)$ is a Riesz basis for $L^{2}(\mathbf{R})$, then $D^{-}(\Lambda)=D^{+}(\Lambda)=1$.

(c) If $D^{-}(\Lambda)<1$, then $\mathcal{G}(g, \Lambda)$ is not a frame for $L^{2}(\mathbf{R})$.

The result above was first proved (under some extra hypotheses) by Landau [91] and Ramanathan and Steger [103]. Inspired by the paper [60], those extra hypotheses were removed in [32] (and the result was also extended to higher dimensions and multiple generators). The papers [8], [9] show among other results that Theorem 9.14 is not just a result about Gabor frames but can be extended to the much more general situation of localized frames. Moreover, new consequences follow even for Gabor frames, such as the fact that the index set of any tight Gabor frame must possess a certain amount of uniformity, in the sense that the upper and lower Beurling densities will coincide, i.e., $D^{-}(\Lambda)=D^{+}(\Lambda)$. Related results appear in [6], [7]. Localized frames were independently introduced by Gröchenig in [58]; among other results Gröchenig shows that localized frames are frames not merely for the underlying Hilbert space $H$ but also for an entire family of associated Banach spaces. Additional papers on localized frames are [57], [36].

Since the Beurling density of a rectangular lattice is $D^{-}(\alpha \mathbf{Z} \times \beta \mathbf{Z})=$ $D^{+}(\alpha \mathbf{Z} \times \beta \mathbf{Z})=\frac{1}{\alpha \beta}$, Theorem 9.14 almost, but not quite, recovers Theorem 9.13. One trivial difference is in part (b) of the two theorems: the implications proceed in both directions in Theorem 9.13(b) but only in one direction in Theorem 9.14(b). For a counterexample to the converse direction in Theorem 9.14(b), take a Gabor frame $\mathcal{G}(g, \Lambda)$ that happens to be a Riesz basis and add a single point, say $\lambda$, to $\Lambda$. Then $\mathcal{G}(g, \Lambda \cup\{\lambda\})$ is a redundant frame, but the Beurling density is the same, $D^{-}(\Lambda \cup\{\lambda\})=D^{+}(\Lambda \cup\{\lambda\})=1$.

On the other hand, the difference between Theorem 9.13(c) and Theorem 9.14(c) is much more significant. Ramanathan and Steger conjectured in [103] that Theorem 9.14(c) should be improvable to say that if $D^{-}(\Lambda)<1$ then $\mathcal{G}(g, \Lambda)$ is incomplete in $L^{2}(\mathbf{R})$, but this was shown in [17] to be false: for any $\varepsilon>0$ there exists a function $g \in L^{2}(\mathbf{R})$ and a set $\Lambda \subset \mathbf{R}^{2}$ with 
$D^{+}(\Lambda)<\varepsilon$ such that $\mathcal{G}(g, \Lambda)$ is complete. The counterexample built in a fundamental way on the work of Landau on the completeness of exponentials in $L^{2}(S)$ where $S$ is a finite union of intervals. Another counterexample, in which $\Lambda$ is a subset of a lattice, appears in [113]. In [99], [100], it is shown that there exist $g \in L^{2}(\mathbf{R})$ and $\Lambda$ of the form $\Lambda=\left\{\left(\lambda_{n}, 0\right)\right\}_{n \in \mathbf{Z}}$ such that $\mathcal{G}(g, \Lambda)=\left\{T_{\lambda_{n}} g\right\}_{n \in \mathbf{Z}}$ is complete in $L^{2}(\mathbf{R})$ and $\left\{\lambda_{n}\right\}_{n \in \mathbf{Z}}$ is a perturbation of the integers $\mathbf{Z}$. Thus $D^{+}(\Lambda)=0$ for this example.

We close this section with some remarks.

Remark 9.15. a. Theorems 9.13 and 9.14 provide necessary conditions for a Gabor system to be a frame. Some sufficient conditions are known; see for example [41, Sec. 3.4], [55, Sec. 6.5], [71, Sec. 4.1]. For the Gaussian function $g(x)=e^{-x^{2}}$, there is actually a complete characterization of when $\mathcal{G}\left(e^{-x^{2}}, \alpha \mathbf{Z} \times \beta \mathbf{Z}\right)$ is a frame; specifically, this occurs if and only if $\alpha \beta<1$; see the simple proof and additional references in [79].

The Gaussian is one of only three functions for which such a characterization is currently known (not counting trivial modifications such as translations, modulations, dilations). The other two are the hyperbolic secant $g(x)=\frac{1}{\cosh \pi x}$ and the two-sided exponential $g(x)=e^{-|x|}$ [83], [84]. The precise set of $(\alpha, \beta)$ for which $\mathcal{G}\left(\chi_{[0,1)}, \alpha \mathbf{Z} \times \beta \mathbf{Z}\right)$ is a frame is not known, but surprisingly, it appears to be an extremely complicated set, called "Janssen's tie" [82]. The problem of characterizing those sets $E \subset \mathbf{R}$ such that $\mathcal{G}\left(\chi_{E}, \mathbf{Z}^{2}\right)$ is a frame has been shown to be equivalent to a longstanding open problem of Littlewood [25].

b. In concrete situations, a given Hilbert space often does not appear in isolation, but is associated with an extended family of Banach spaces. A classical example is $L^{2}(\mathbf{R})$ sitting inside the class of Lebesgue spaces $L^{p}(\mathbf{R})$, which are themselves contained in the extended family of Besov spaces $B_{s}^{p, q}(\mathbf{R})$ and Triebel-Lizorkin spaces $F_{s}^{p, q}(\mathbf{R})$. Wavelet frames (briefly discussed in Section 9.4.3) typically provide expansions of functions not only in the Hilbert space $L^{2}(\mathbf{R})$ but in all of the Besov and Triebel-Lizorkin spaces simultaneously. The norms of these spaces are related to smoothness properties of the function, and this smoothness information can likewise be identified by examining the coefficients in the wavelet expansion. Analogously, Gabor frames can provide expansions not only for $L^{2}(\mathbf{R})$ but for an appropriate extended family of Banach spaces $M_{s}^{p, q}(\mathbf{R})$ known as the modulation spaces. The modulation space norms quantify time-frequency concentration rather than smoothness. These spaces were introduced and extensively studied by Feichtinger and Gröchenig. An excellent textbook development of the modulation spaces appears in [55, Chs. 11-14]. We also mention the mostly expository paper [66], which surveys one application of the modulation spaces to the analysis of spectral properties of integral operators. 


\subsubsection{Gabor Riesz Bases and the Balian-Low Theorem}

In this section we consider Gabor frames that are Riesz bases. At least for the case where $\Lambda$ is a rectangular lattice, all Gabor Riesz bases are "bad." Paraphrased in a qualitative form, the Balian-Low Theorem or BLT ${ }^{4}$ states that if $\mathcal{G}(g, \alpha \mathbf{Z} \times \beta \mathbf{Z})$ is a Gabor Riesz basis (which by Theorem 9.13 can only happen when $\alpha \beta=1$ ), then $g$ is either not smooth or decays poorly at infinity. Here are two precise variations on this theme. In these, $\hat{g}$ denotes the Fourier transform of $g$ (we use Benedetto's preferred normalization $\hat{g}(\omega)=$ $\left.\int g(x) e^{-2 \pi i \omega x} d x\right)$.

\section{Theorem 9.16 (Balian-Low Theorems).}

(a) Classical BLT: If $g \in L^{2}(\mathbf{R})$ is such that $\mathcal{G}(g, \alpha \mathbf{Z} \times \beta \mathbf{Z})$ is a Gabor Riesz basis for $L^{2}(\mathbf{R})$, then

$$
\left(\int_{-\infty}^{\infty}|\operatorname{tg}(t)|^{2} d t\right)\left(\int_{-\infty}^{\infty}|\omega \hat{g}(\omega)|^{2} d \omega\right)=\infty
$$

(b) Amalgam BLT: If $g \in L^{2}(\mathbf{R})$ is such that $\mathcal{G}(g, \alpha \mathbf{Z} \times \beta \mathbf{Z})$ is a Gabor Riesz basis for $L^{2}(\mathbf{R})$, then $g, \hat{g} \notin W\left(C_{0}, \ell^{1}\right)$, where

$$
W\left(C_{0}, \ell^{1}\right)=\left\{\text { continuous } f: \sum_{k=-\infty}^{\infty}\left\|f \cdot \chi_{[k, k+1]}\right\|_{\infty}<\infty\right\} \text {. }
$$

Note that the quantity appearing on the left-hand side of (9.7) is the Heisenberg product that appears in the Classical Uncertainty Principle. In particular, the generator of any Gabor Riesz basis must maximize uncertainty. See [50] for a general survey on the uncertainty principle, [12] for some other connections between Gabor systems and uncertainty principles, and [56] for new uncertainty principles in time-frequency analysis.

The Classical BLT was introduced independently by Balian [10] and Low [97]. A gap in their proofs was later filled in Daubechies' influential article [40], which also contains many important results on frames, Gabor systems, and wavelets (much of which was incorporated into [41]). An exquisite proof of the Classical BLT for the case of orthonormal bases was given by Battle [11], based on the operator theory associated with the Classical Uncertainty Principle; this proof was extended to Riesz bases in [43]. Variations on these proofs that avoid the use of distributional differentiation are given in [17]. A survey of recent results on the BLT appears in this volume [38].

\footnotetext{
${ }^{4}$ Yes, that is a joke: in the United States a "BLT" is a Bacon, Lettuce, and Tomato sandwich. As far as I know, this acronym was first used in print in [17], due entirely to John Benedetto's wonderful sense of humor.
} 
The Amalgam BLT was first published in [17], although it was proved earlier in [63]. It is shown in [17] that neither version of the BLT implies the other. The space $W\left(C_{0}, \ell^{1}\right)$ appearing in (9.8) is an example of a Wiener amalgam space. While specific amalgam spaces have been used often throughout the mathematical literature, Feichtinger introduced and extensively studied general amalgam spaces, whose norm combines a local criterion for membership with a global criterion. The article [65] is an expository introduction to these spaces, including many references as well as a simple proof of the Amalgam BLT.

The BLT emphasizes that for applications where Gabor systems are useful, it is redundant Gabor frames that will usually be most appropriate. On the other hand, there is a remarkable construction known as Wilson bases that are in the spirit of time-frequency constructions, are generated by "nice" functions, and are unconditional bases not only for $L^{2}(\mathbf{R})$ but also for the class of modulation spaces. Unfortunately, there is a cost in the form of increased technicality; Wilson bases do not have the simple form that Gabor systems have. For more on Wilson bases, we suggest [55, Sec. 8].

Let us close this section by pointing out some related open problems and questions.

- Does the BLT hold for lattices that are nonrectangular? This question was recently answered affirmatively for the case of the Classical BLT in one dimension in [59], but as soon as we move to higher dimensions, only partial results are known. In particular, it is shown in [59] that the Classical BLT generalizes to the case of symplectic lattices in higher dimensions, but for nonsymplectic lattices little is known (see also Section 9.5.1 below). Some weaker partial results are also known to hold for the analogue of the Classical BLT for irregular Gabor Riesz bases. For more on the BLT on symplectic lattices, see [59] and [15], and for other recent results on the BLT see [14], [53].

- The situation for the Amalgam BLT is even less clear: it is not known even in one dimension if the Amalgam BLT still holds if rectangular lattices are replaced by general lattices $A\left(\mathbf{Z}^{2}\right)$ or by irregular sets of time-frequency shifts.

- Little is known about Gabor systems that are Schauder bases but not Riesz bases for $L^{2}(\mathbf{R})$. One such example is $\mathcal{G}\left(g, \mathbf{Z}^{2}\right)$, where $g(x)=$ $|x|^{\alpha} \chi_{\left[-\frac{1}{2}, \frac{1}{2}\right]}(x)$ and $0<\alpha<\frac{1}{2}$ (compare Example 9.10). It is not known if the BLT theorems hold if Gabor Riesz bases are replaced by Gabor Schauder bases, although it is interesting to note that the proof of the Weak BLT given in [17, Thm. 7.4] or [59, Thm. 8] generalizes from Riesz bases to Schauder bases.

- It was conjectured in [45] that Gabor Schauder bases follow the same Nyquist-type rules as Gabor Riesz bases, i.e., if $\mathcal{G}(g, \Lambda)$ is a Gabor Schauder basis then $D^{-}(\Lambda)=D^{+}(\Lambda)=1$. Some partial results were obtained in 
[45], but the conjecture remains open. More generally, is there a Nyquist density result for Gabor systems that are minimal and complete but not bases, such as $\mathcal{G}\left(e^{-x^{2}}, \mathbf{Z}^{2} \backslash(0,0)\right)$ from Example 9.12?

- In [115], [116], Zalik gave some necessary and some sufficient conditions on $g \in L^{2}(\mathbf{R})$ and countable subsets $\Gamma \subset \mathbf{R}$ such that $\left\{T_{a} g\right\}_{a \in \Gamma}$ is complete in $L^{2}(\mathbf{R})$; see also the recent constructions in [99], [100]. Olson and Zalik proved in [101] that no such system of pure translations can be a Riesz basis for $L^{2}(\mathbf{R})$, and conjectured that no such system can be a Schauder basis. This conjecture is still open. Since $\left\{T_{a} g\right\}_{a \in \Gamma}=\mathcal{G}(g, \Gamma \times\{0\})$ and $D^{-}(\Gamma \times\{0\})=0$, it follows from Theorem 9.14 that such a system can never be a frame for $L^{2}(\mathbf{R})$. Similarly, if the density theorem conjectured for Gabor Schauder bases in the preceding question could be proved, then the Olson-Zalik Conjecture would follow as a corollary.

\subsubsection{The Zero Divisor Conjecture and a Contrast to Wavelets}

One motivation for the HRT Conjecture is simply the question of how similar Gabor frames are to the system of exponentials presented in Example 9.5: Is every Gabor frame finitely linearly independent? Since this is a question about finite subsets, it leads directly to the statement of Conjecture 9.1.

As one motivation for why we might be interested in such a question, let us contrast the situation for a different class of objects, wavelet systems. For more background on wavelets, we suggest [41], [74], or, for more elementary introductions, [111], [19]. Many of the influential early wavelet papers and their precursors are reprinted in [72].

Just as Gabor systems are associated with the Schrödinger representation of the Heisenberg group, wavelets are associated with a representation of another group, the affine or $a x+b$ group. Instead of considering time-frequency shifts, consider time-scale shifts, which are compositions of the translation $T_{a} g(x)=g(x-a)$ with the dilation

$$
D_{r} g(x)=r^{1 / 2} g(r x),
$$

which has been normalized so that $D_{r}$ is a unitary operator on $L^{2}(\mathbf{R})$. Specifically, given $g \in L^{2}(\mathbf{R})$ and a sequence $\Lambda \subset \mathbf{R} \times \mathbf{R}^{+}$, the wavelet system generated by $g$ and $\Lambda$ is the collection of time-scale shifts

$$
\mathcal{W}(g, \Lambda)=\left\{T_{a} D_{b} g\right\}_{(a, b) \in \Lambda} .
$$

The group underlying wavelet systems is the affine group, which is the set $\mathbf{A}=$ $\mathbf{R} \times \mathbf{R}^{+}$endowed with a group multiplication that makes the mapping $(a, b) \mapsto$ $T_{a} D_{b}$ an injective homomorphism of $\mathbf{A}$ into the set of unitary mappings of $L^{2}(\mathbf{R})$ onto itself, and this mapping is the corresponding representation.

For wavelet systems, the analogue of Conjecture 9.1 fails. For example, a function $\varphi$ is said to be refinable, or is called a scaling function, if there exist coefficients $c_{k}$ such that 


$$
\varphi(x)=\sum_{k=-\infty}^{\infty} c_{k} \varphi(2 x-k)
$$

(often the term scaling function is reserved for functions that satisfy additional requirements beyond just (9.9), for example, they may be required to be associated with a multiresolution analysis, see Remark 9.17 below). If only finitely many coefficients $c_{k}$ in (9.9) are nonzero, then we have an expression of finite linear dependence among the time-scale shifts of $\varphi$. That is, $\mathcal{W}(\varphi, \Lambda)$ is dependent with a finite $\Lambda$. It is not hard to construct such functions, for example, the box function $b=\chi_{[0,1)}$ satisfies the refinement equation

$$
b(x)=b(2 x)+b(2 x-1) .
$$

Thus the analogue of Conjecture 9.1 fails when the Heisenberg group is replaced by the affine group. This raises the fundamental question: what is the basic difference between the affine group and the Heisenberg group which makes their behavior with regard to this conjecture so different? While both the affine group and the Heisenberg group are non-Abelian, the Heisenberg group is "nearly Abelian" in contrast to the affine group. For example, both are locally compact topological groups, and the Heisenberg group is unimodular (left and right Haar measure coincide), as are all Abelian locally compact groups, whereas the affine group is nonunimodular (see [71]). Another difference is that the Heisenberg group has discrete subgroups (e.g., $\{1\} \times \mathbf{Z} \times \mathbf{Z}$ ) while the affine group does not. Which, if any, of these are the essential difference in regard to the HRT Conjecture?

More generally, we could replace the Heisenberg or affine groups by other groups, and consider representations of arbitrary topological groups on $L^{2}(\mathbf{R})$. This leads to a connection with the open Zero Divisor Conjecture in abstract algebra, introduced in [75]. For a discussion of the Zero Divisor Conjecture and the connection between zero divisors and independence of translates we refer to the papers by Linnell, including [94], [95], [96].

We conclude this section by pointing out a few other related questions and connections.

Remark 9.17. a. Refinable functions play a central role in several areas, including subdivision schemes in computer-aided geometric design and the construction of orthonormal wavelet bases [41]. The now-classical method for constructing a wavelet orthonormal basis for $L^{2}(\mathbf{R})$ begins with a scaling function $\varphi$ which has the additional property that the collection of integer translates $\{\varphi(x-k)\}_{k \in \mathbf{Z}}$ is an orthonormal sequence in $L^{2}(\mathbf{R})$. Such a $\varphi$ leads to a multiresolution analysis (MRA) for $L^{2}(\mathbf{R})$, from which is deduced the existence of a wavelet $\psi$ which has the property that $\left\{2^{n / 2} \psi\left(2^{n} x-k\right)\right\}_{n, k \in \mathbf{Z}}$ is an orthonormal basis for $L^{2}(\mathbf{R})$. Thus the scaling function $\varphi$, whose time-scale translates are finitely dependent, leads to another function $\psi$ which generates an orthonormal basis for $L^{2}(\mathbf{R})$ via time-scale translates. 
b. There are also non-MRA constructions of orthonormal wavelet bases; these are especially surprising in higher dimensions [5], [18], [39].

c. The refinement equation (9.9) implies that the graph of a refinable function $\varphi$ possesses some self-similarity. This leads to connections to fractals and iterated function systems. The analysis of the properties of refinable functions is an interesting topic with a vast literature; we refer to the paper [21] for references.

d. While the analogue of the HRT Conjecture fails in general, Christensen and Lindner [34], [35] have interesting partial results on when independence holds, including estimates of the frame bounds of finite sets of time-frequency or time-scale shifts.

e. There are useful recent characterizations of frames that apply in both the wavelet and Gabor settings by Hernández, Labate, and Weiss [90], [73].

f. One fundamental difference between wavelet orthonormal bases or Riesz bases and their Gabor analogues is that the analogue of the Balian-Low Theorems fail for wavelets. For example, it is possible to find Schwartz-class functions $\psi$, or compactly supported $\psi \in C^{(n)}(\mathbf{R})$ with $n$ arbitrarily large, which generate wavelet orthonormal bases for $L^{2}(\mathbf{R})$. Another fundamental difference occurs in regard to density phenomenon. While Theorem 9.14 shows that Gabor frames have a Nyquist density similar to the one obeyed by the system of exponentials discussed in Example 9.5, there is no exact analogue of the Nyquist density for wavelet frames. Analogues of the Beurling density appropriate for the affine group were introduced in the papers [67], [110] (see also [89] for a comparison of these definitions and [37] for density conditions on combined Gabor/wavelet systems). While there is no Nyquist phenomenon in the sense that wavelet frames can be constructed with any particular density, it was shown that wavelet frames cannot have zero or infinite density, and the details of the arguments suggest a surprising amount of similarity between the Heisenberg and affine cases, cf. [68].

\subsection{Partial Results on the HRT Conjecture}

Now we return to the HRT Conjecture itself, and present some of the partial results that are known and some of the techniques used to obtain them. Using the notation introduced so far, we can reword the conjecture as follows:

- If $g \in L^{2}(\mathbf{R})$ is nonzero and $\Lambda=\left\{\left(\alpha_{k}, \beta_{k}\right)\right\}_{k=1}^{N}$ is a finite set of distinct points in $\mathbf{R}^{2}$, then $\mathcal{G}(g, \Lambda)$ is linearly independent.

Before presenting the partial results themselves, let us make some general remarks on why the conjecture seems to be difficult. A profoundly deep explanation would be an interesting research problem in itself; we will only point out some particular difficulties. 
One major problem is that the conjecture is resistant to transform techniques. For example, applying the Fourier transform simply interchanges the translations and modulations, converting the problem into one of exactly the same type. Another natural transform for time-frequency analysis is the shorttime Fourier transform (STFT). Given a "window function" $\varphi \in L^{2}(\mathbf{R})$, the STFT of $g \in L^{2}(\mathbf{R})$ with respect to $\varphi$ is

$$
V_{\varphi} g(x, \omega)=\left\langle g, M_{\omega} T_{x} \varphi\right\rangle, \quad(x, \omega) \in \mathbf{R}^{2} .
$$

With $\varphi$ fixed, the mapping $g \mapsto V_{\varphi} g$ is an isometry of $L^{2}(\mathbf{R})$ into $L^{2}\left(\mathbf{R}^{2}\right)$. However,

$$
V_{\varphi}\left(M_{b} T_{a} g\right)(x, \omega)=e^{-2 \pi i a b \omega} V_{\varphi} g(x-a, \omega-b) .
$$

Thus, the STFT converts translations and modulations of $g$ into two-dimensional translations and modulations of $V_{\varphi} g$, again yielding a problem of the same type, except now in two dimensions. There are many closely related transforms, such as the Wigner distribution, and quadratic versions of these transforms such as $g \mapsto V_{g} g$, but all of these have related difficulties. On the other hand, as we will see below, for special cases transforms can yield useful simplifications.

\subsubsection{Linear Transformations of the Time-Frequency Plane}

There is a class of transformations that we can apply that will sometimes simplify the geometry of the set of points $\Lambda=\left\{\left(\alpha_{k}, \beta_{k}\right)\right\}_{k=1}^{N}$ appearing in Conjecture 9.1. For example, let $A$ be the linear transformation $A(a, b)=$ $\left(\frac{a}{r}, b r\right)$ where $r>0$ (note that $\left.\operatorname{det}(A)=1\right)$. Then

$$
D_{r}\left(M_{\beta_{k}} T_{\alpha_{k}} g\right)(x)=r^{1 / 2} e^{2 \pi i \beta_{k} r x} g\left(r x-\alpha_{k}\right)=M_{\beta_{k} r} T_{\frac{\alpha_{k}}{r}}\left(D_{r} g\right)(x) .
$$

Since the dilation $D_{r}$ is a unitary map, it preserves independence, and hence

$$
\mathcal{G}(g, \Lambda) \text { is independent } \Longleftrightarrow \mathcal{G}\left(D_{r} g, A(\Lambda)\right) \text { is independent. }
$$

Thus, we can change the configuration of the points from the set $\Lambda$ to the set $A(\Lambda)$ at the cost of replacing the function $g$ by a dilation of $g$.

In fact, if $A$ is any linear transformation of $\mathbf{R}^{2}$ onto itself with $\operatorname{det}(A)=1$, then there exists a unitary transformation $U_{A}: L^{2}(\mathbf{R}) \rightarrow L^{2}(\mathbf{R})$ such that

$$
U_{A}(\mathcal{G}(g, \Lambda))=\left\{U_{A}\left(M_{b} T_{a} g\right)\right\}_{(a, b) \in \Lambda}=\left\{c_{A}(a, b) M_{v} T_{u}\left(U_{A} g\right)\right\}_{(u, v) \in A(\Lambda)},
$$

where $c_{A}(a, b)$ are constants of unit modulus determined by $A$. The rightmost set in (9.10) is not a Gabor system, but is obtained from the Gabor system $\mathcal{G}\left(U_{A} g, A(\Lambda)\right)$ by multiplying each element by a constant of unit modulus. Such multiplications do not change the two properties we are interested in here, namely, being a frame or being linearly independent. In particular, 
$\mathcal{G}(g, \Lambda)$ is independent $\Longleftrightarrow \mathcal{G}\left(U_{A} g, A(\Lambda)\right)$ is independent.

As a specific example, consider the shear $A(a, b)=(a, b+r a)$. Then $U_{A}$ is the modulation by a "chirp" given by $U_{A} f(x)=e^{\pi i r x^{2}} f(x)$. To see this, set $c_{A}(a, b)=e^{-\pi i r a^{2}}$ and verify that

$$
c_{A}(a, b) M_{b+r a} T_{a}\left(U_{A} g\right)(x)=U_{A}\left(M_{b} T_{a} g\right)(x) .
$$

Another example is $A(a, b)=(b,-a)$, rotation by $\pi / 2$, for which $U_{A}=\mathcal{F}$, the Fourier transform.

In addition to linear transformations of $\mathbf{R}^{2}$, we can also use rigid translations, for they correspond to replacing $g$ by $M_{d} T_{c} g$ and $\Lambda$ by $\Lambda-(c, d)$.

The operators $U_{A}$ are called metaplectic transforms. Every linear transformation $A$ of $\mathbf{R}^{2}$ with determinant 1, i.e., every element of the special linear group $S L(2, \mathbf{R})$, yields a metaplectic transform. Unfortunately, as soon as we move to higher dimensions, this is no longer true. Only matrices lying in the symplectic group $S_{p}(d)$, which is a subset of $S L(2 d, \mathbf{R})$, yield metaplectic transforms (the symplectic group consists of those invertible matrices $A$ which preserve the symplectic form $\left[z, z^{\prime}\right]=x^{\prime} \cdot \omega-x \cdot \omega^{\prime}$, where $z=(x, \omega)$, $\left.z^{\prime}=\left(x^{\prime}, \omega^{\prime}\right) \in \mathbf{R}^{2 d}\right)$. This stems from properties of the Heisenberg group, and for more details we refer to [55, Ch. 9]. In any case, this means that some of the simplifications we apply below to prove some special cases of the HRT Conjecture may not apply in higher dimensions.

\subsubsection{Special Case: Points on a Line}

In this section we will prove a special case of the HRT Conjecture, assuming that $\Lambda=\left\{\left(\alpha_{k}, \beta_{k}\right)\right\}_{k=1}^{N}$ is a set of collinear points. By applying an appropriate metaplectic transform, we may assume that $\Lambda=\left\{\left(\alpha_{k}, 0\right)\right\}_{k=1}^{N}$. Then $\mathcal{G}(g, \Lambda)=$ $\left\{g\left(x-\alpha_{k}\right)\right\}_{k=1}^{N}$ is a finite set of translations of $g$. Suppose that we have $\sum_{k=1}^{N} c_{k} g\left(x-\alpha_{k}\right)=0$ a.e. for some scalars $c_{1}, \ldots, c_{N}$. Taking the Fourier transform of both sides of this equation converts translations to modulations, resulting in the equation

$$
\sum_{k=1}^{N} c_{k} e^{-2 \pi i \alpha_{k} \xi} \hat{g}(\xi)=0 \text { a.e. }
$$

Since $\hat{g} \neq 0$, it is nonzero on some set of positive measure. But then the nonharmonic trigonometric polynomial $m(\xi)=\sum_{k=1}^{N} c_{k} e^{-2 \pi i \alpha_{k} \xi}$ must be zero on a set of positive measure. If the $\alpha_{k}$ are integer, it follows immediately from the Fundamental Theorem of Algebra that $c_{1}=\cdots=c_{N}=0$. This is still true for arbitrary $\alpha_{k}$, since $m$ can be extended from real values of $\xi$ to complex values, and the extension is an analytic function. Thus, we conclude that $\mathcal{G}(g, \Lambda)$ is linearly independent when the points in $\Lambda$ are collinear.

While the HRT Conjecture turns out to be trivial when we restrict to the case of pure translations of $g$, there are still many interesting remarks to 
be made about this case. Consider first the fact that if we replace $L^{2}(\mathbf{R})$ by $L^{\infty}(\mathbf{R})$ then finite sets of translations can be linearly dependent (for example, consider any periodic function). In one dimension, it can be shown that any finite set of translations of a nonzero function $g \in L^{p}(\mathbf{R})$ with $p<\infty$ are independent, but note that this is already a more difficult problem because the Fourier transform exists only distributionally when $p>2$. Moreover, Rosenblatt and Edgar have shown that there is a surprise as soon as we move to higher dimensions: sets of translates can be dependent for finite $p$. The following result was proved in [48], [105].

\section{Theorem 9.18.}

(a) If $g \in L^{p}\left(\mathbf{R}^{d}\right)$ is nonzero and $1 \leq p \leq \frac{2 d}{d-1}, p \neq \infty$, then $\left\{g\left(x-\alpha_{k}\right)\right\}_{k=1}^{N}$ is linearly independent for any finite set of distinct points $\left\{\alpha_{k}\right\}_{k=1}^{N}$ in $\mathbf{R}^{d}$.

(b) If $\frac{2 d}{d-1}<p \leq \infty$, then there exists a nonzero $g \in L^{p}\left(\mathbf{R}^{d}\right)$ and distinct points $\left\{\alpha_{k}\right\}_{k=1}^{N}$ in $\mathbf{R}^{d}$ such that $\left\{g\left(x-\alpha_{k}\right)\right\}_{k=1}^{N}$ is linearly dependent.

We close this section by noting that subspaces of $L^{2}(\mathbf{R})$ of the form $V=\overline{\operatorname{span}}\left(\left\{T_{\alpha_{k}} g\right\}_{k \in \mathbf{N}}\right)$ generated from translations of a given function are important in a wide variety of applications. In particular, a subspace of the form $V=\overline{\operatorname{span}}\left(\left\{T_{k} g\right\}_{k \in \mathbf{N}}\right)$ is invariant under integer translations, and hence is called a shift-invariant space (but it need not be translation-invariant, which means invariant under all translations). Shift-invariant spaces play key roles in sampling theory, the construction of wavelet bases and frames, and other areas. For a recent research-survey of shift-invariant spaces in sampling theory, we suggest [2].

\subsubsection{Special Case: Lattices}

Suppose that $\Lambda=\left\{\left(\alpha_{k}, \beta_{k}\right)\right\}_{k=1}^{N}$ is a finite subset of some lattice $A\left(\mathbf{Z}^{2}\right)$, where $A$ is an invertible matrix. By applying a metaplectic transform, we may assume that $\Lambda$ is a subset of a rectangular lattice $\alpha \mathbf{Z} \times \beta \mathbf{Z}$ with $\alpha \beta=|\operatorname{det}(A)|$. We say that it is a unit lattice if $\alpha \beta=1$; in this case, by applying a dilation we can assume $\alpha=\beta=1$. The HRT Conjecture is easily settled for this special case by applying the Zak transform, which is the unitary mapping of $L^{2}(\mathbf{R})$ onto $L^{2}\left([0,1)^{2}\right)$ given by

$$
Z f(t, \omega)=\sum_{k \in \mathbf{Z}} f(t-k) e^{2 \pi i k \omega}, \quad(t, \omega) \in[0,1)^{2},
$$

(the series converges in the norm of $L^{2}\left([0,1)^{2}\right)$ ). The Zak transform was first introduced by Gelfand (see [55, p. 148]), and goes by several names, including the Weil-Brezin map (representation theory and abstract harmonic analysis) and $k-q$ transform (quantum mechanics). For more information, we refer to Janssen's influential paper [77] and survey [78], or Gröchenig's text [55]. For our purposes, the most important property of the Zak transform is that 


$$
Z\left(M_{k} T_{n} g\right)(t, \omega)=e^{2 \pi i n t} e^{-2 \pi i k \omega} Z g(t, \omega), \quad(k, n) \in \mathbf{Z}^{2} .
$$

It follows easily from this that any finite linear combination of functions $M_{k} T_{n} g$ with $(k, n) \in \mathbf{Z}^{2}$ is independent, using the fact that a nontrivial (two-dimensional) trigonometric polynomial cannot vanish on a set of positive measure. This settles the case $\alpha \beta=1$.

This argument cannot be extended to more general rectangular lattices $\alpha \mathbf{Z} \times \beta \mathbf{Z}$ with $\alpha \beta \neq 1$ because $Z\left(M_{k \beta} T_{n \alpha} g\right)$ is no longer just a two-dimensional exponential times $Z g$. A fundamental obstacle is that the operators $M_{k}, T_{n}$ commute when $k, n$ are integers, but the operators $M_{k \beta}, T_{n \alpha}$ do not commute in general. On the other hand, the operators $M_{\beta}, T_{\alpha}$ generate a von Neumann algebra, and it is through this connection that Linnell was able to prove the HRT Conjecture for the special case that $\Lambda$ is contained in an arbitrary lattice [96].

Although this is contained in Linnell's result, let us sketch a proof of the HRT Conjecture for the special case $N=3$, since it reveals one of the difficulties in trying to prove the general case. If $N=3$ then, by applying a metaplectic transform, we may assume that $\Lambda=\{(0,0),(a, 0),(0,1)\}$, and hence $\mathcal{G}(g, \Lambda)=\left\{g(x), g(x-a), e^{2 \pi i x} g(x)\right\}$. Suppose that

$$
c_{1} g(x)+c_{2} g(x-a)+c_{3} e^{2 \pi i x} g(x)=0 \text { a.e. }
$$

If any one of $c_{1}, c_{2}, c_{3}$ is zero, then we are back to the collinear case, so we may assume they are all nonzero. Rearranging, we obtain

$$
g(x-a)=m(x) g(x) \text { a.e., }
$$

where $m(x)=-\frac{1}{c_{2}}\left(c_{1}+c_{3} e^{2 \pi i x}\right)$. Note that $m$ is 1-periodic. Iterating (9.11), we obtain for integer $n>0$ that

$$
|g(x-n a)|=|g(x)| \prod_{j=0}^{n-1}|m(x-j a)|=|g(x)| e^{n \cdot \frac{1}{n} \sum_{j=0}^{n-1} p(x-j a)} \text { a.e. }
$$

where $p(x)=\ln |m(x)|$. Now, $p$ is 1-periodic, so if $a$ is irrational then the points $\{x-j a \bmod 1\}_{j=0}^{\infty}$ are a dense subset of $[0,1)$. In fact, they are "well distributed" in a technical sense due to the fact that $x \mapsto x+a \bmod 1$ is an ergodic mapping of $[0,1)$ onto itself (ergodic means that any subset of $[0,1$ ) which is invariant under this map must either have measure 0 or 1$)$. Hence the quantity $\frac{1}{n} \sum_{j=0}^{n-1} p(x-j a)$ is like a Riemann sum approximation to $\int_{0}^{1} p(x) d x$, except that the rectangles with height $p(x-j a)$ and width $\frac{1}{n}$ are distributed "randomly" around $[0,1)$ instead of uniformly, possibly even with overlaps or gaps. Still, the ergodicity ensures that the Riemann sum analogy is a good one in the limit: the Birkhoff Ergodic Theorem implies that

$$
\lim _{n \rightarrow \infty} \frac{1}{n} \sum_{j=0}^{n-1} p(x-j a)=\int_{0}^{1} p(x) d x=C \text { a.e. }
$$


To be sure, we must verify that $C=\int_{0}^{1} p(x) d x$ exists and is finite, but this can be shown based on the fact that any singularities of $p$ correspond to zeros of the well-behaved function $m$. For more information on ergodic theory, we refer to [112].

Thus from (9.11) and (9.13) we see that $|g(x-n a)| \approx e^{C n}|g(x)|$. More precisely, if we fix $\varepsilon>0$, then $\frac{1}{n} \sum_{j=0}^{n-1} p(x-j a) \geq(C-\varepsilon)$ for $n$ large enough. Let us ignore the fact that "large enough" depends on $x$ (or, by applying Egoroff's Theorem, restrict to a subset where the convergence in (9.13) is uniform). Substituting into (9.12) then yields $|g(x-n a)| \geq e^{(C-\varepsilon) n}|g(x)|$ for $n$ large. Considering $x$ in a set of positive measure where $g$ is nonzero and the fact that $g \in L^{2}(\mathbf{R})$, we conclude that $C-\varepsilon<0$, and hence $C \leq 0$. A converse argument, based on the relation $g(x)=m(x+a) g(x+a)$ similarly yields the fact that $C \geq 0$. This still allows the possibility that $C=0$, but a slightly more subtle argument presented in [69] also based on ergodicity yields the full result. The case for $a$ rational is more straightforward, since in this case the points $x-j a$ mod 1 repeat themselves.

The argument given above can be extended slightly: we could take $\Lambda=$ $\{(0, j)\}_{j=0}^{N} \cup\{(a, 0)\}$, for then we would still have (9.11) holding, with a different, but still 1-periodic, function $m$. However, the periodicity is critical in order to apply ergodic theory as we have done. An additional fundamental difficulty to extending further is that as soon as we have more than two distinct translates, we cannot rearrange a dependency relation into a form similar to (9.11) that can be easily iterated. For example, with three distinct sets of translations, instead of (9.11) we would have an equation like $g(x-a)=m(x) g(x)+k(x) g(x-b)$, which becomes extremely complicated to iterate.

\subsubsection{Special Case: Compactly Supported Functions}

Choose any finite set $\Lambda \subset \mathbf{R}^{2}$, and suppose that $g \in L^{2}(\mathbf{R})$ is compactly supported, or even just supported on a half-line. Given an arbitrary finite set $\Lambda$, write $\Lambda=\left\{\left(\alpha_{k}, \beta_{k, j}\right)\right\}_{j=1, \ldots, M_{k}, k=1, \ldots, N}$, i.e., for each distinct translate group the corresponding modulates together. Given scalars $c_{k, j}$, suppose

$$
0=\sum_{k=1}^{N} \sum_{j=1}^{M_{k}} c_{k, j} M_{\beta_{k, j}} T_{\alpha_{k}} g(x)=\sum_{k=1}^{N} m_{k}(x) g\left(x-\alpha_{k}\right) \text { a.e., }
$$

where $m_{k}(x)=\sum_{j=1}^{M_{k}} c_{k, j} e^{2 \pi i \beta_{k, j} x}$. Since $g$ is supported in a half-line, the supports of the functions $g\left(x-\alpha_{k}\right)$ overlap some places but not others. If we choose $x$ in the appropriate interval then only one $g\left(x-\alpha_{k}\right)$ can be nonzero. For such $x$, the right-hand side of equation (9.14) will contain only one nonzero term, i.e., it reduces to $m_{k}(x) g\left(x-\alpha_{k}\right)=0$ a.e. for some single $k$. We can find a subset of the support of $g\left(x-\alpha_{k}\right)$ of positive measure for which this is true, which implies the trigonometric polynomial $m_{k}(x)$ vanishes on a set 
of positive measure. But this cannot happen unless $c_{k, j}=0$ for all $j$. We can then repeat this argument to obtain $c_{k, j}=0$ for all $j$ and $k$. For complete details, see [69].

\subsubsection{Special Case: Hermite Functions}

In this section we will prove the HRT Conjecture for the special case that $g(x)=p(x) e^{-x^{2}}$ where $p$ is a nontrivial polynomial. Such functions are finite linear combinations of Hermite functions, and the collection of all such functions is dense in $L^{2}(\mathbf{R})$.

Given an arbitrary finite set $\Lambda$, write $\Lambda=\left\{\left(\alpha_{k}, \beta_{k, j}\right)\right\}_{j=1, \ldots, M_{k}, k=1, \ldots, N}$, with $\alpha_{1}<\cdots<\alpha_{N}$. If $N=1$ then $\Lambda$ is a set of collinear points, so we may assume $N>1$. Given scalars $c_{k, j}$, suppose

$$
\sum_{k=1}^{N} \sum_{j=1}^{M_{k}} c_{k, j} M_{\beta_{k, j}} T_{\alpha_{k}} g(x)=0 \text { a.e. }
$$

Because of the special form of $g$, this simplifies to

$$
e^{-x^{2}} \sum_{k=1}^{N} m_{k}(x) p\left(x-\alpha_{k}\right) e^{2 \alpha_{k} x}=0 \text { a.e. }
$$

where $m_{k}(x)=\sum_{j=1}^{M_{k}} c_{k, j} e^{-\alpha_{k}^{2}} e^{2 \pi i \beta_{k, j} x}$. Without loss of generality we may assume that $m_{1}$ and $m_{N}$ are nontrivial, otherwise ignore those terms and reindex. Then dividing both sides of (9.15) by $e^{-x^{2}} e^{2 \alpha_{N} x}$ and rearranging, we have

$$
m_{N}(x) p\left(x-\alpha_{N}\right)=-\sum_{k=1}^{N-1} m_{k}(x) p\left(x-\alpha_{k}\right) e^{2\left(\alpha_{k}-\alpha_{N}\right) x} \text { a.e. }
$$

However, $\alpha_{k}-\alpha_{N}<0$ for $k=1, \ldots, N-1$, so since each $m_{k}$ is bounded, the right-hand side of (9.16) converges to zero as $x \rightarrow \infty$. On the other hand, as $m_{N}$ is a nontrivial trigonometric polynomial and $p$ is a nontrivial polynomial, the left-hand side does not converge to zero.

\subsubsection{Special Case: Perturbations}

Now that we have proved the HRT Conjecture for dense subsets of $L^{2}(\mathbf{R})$ such as the compactly supported functions, it is tempting to try to prove the general conjecture by applying some form of limiting argument. We will prove next a theorem in this spirit, and then see why this theorem fails to provide a proof of the full conjecture.

We will need to use the following lemma, which is actually just a special case of a general result that characterizes Riesz bases; compare [28, Thm. 3.6.6]. 
Lemma 9.19. Let $\left\{g_{1}, \ldots, g_{N}\right\}$ be a linearly independent set of vectors in a Hilbert space $H$. Let $A, B$ be frame bounds for $\left\{g_{1}, \ldots, g_{N}\right\}$ as a frame for its span $S=\operatorname{span}\left(\left\{g_{1}, \ldots, g_{N}\right\}\right)$. Then

$$
\forall c_{1}, \ldots, c_{N} \in \mathbf{C}, \quad A \sum_{k=1}^{N}\left|c_{k}\right|^{2} \leq\left\|\sum_{k=1}^{N} c_{k} g_{k}\right\|^{2} \leq B \sum_{k=1}^{N}\left|c_{k}\right|^{2} .
$$

Proof. Let $\left\{\tilde{g}_{1}, \ldots, \tilde{g}_{N}\right\}$ be the canonical dual frame for $\left\{g_{1}, \ldots, g_{N}\right\}$ in $S$. Given $c_{1}, \ldots, c_{N}$, set $f=\sum_{k=1}^{N} c_{k} g_{k}$. The frame expansion of $f$ is $f=$ $\sum_{k=1}^{N}\left\langle f, \tilde{g}_{k}\right\rangle g_{k}$, and because of independence we must have $c_{k}=\left\langle f, \tilde{g}_{k}\right\rangle$. Since $\frac{1}{B}, \frac{1}{A}$ are frame bounds for the dual frame $\left\{\tilde{g}_{1}, \ldots, \tilde{g}_{N}\right\}$, we have

$$
\frac{1}{B}\|f\|^{2} \leq \sum_{k=1}^{N}\left|\left\langle f, \tilde{g}_{k}\right\rangle\right|^{2} \leq \frac{1}{A}\|f\|^{2},
$$

and rearranging (9.18) gives (9.17).

We will also need to use the continuity of the operator groups $\left\{T_{x}\right\}_{x \in \mathbf{R}}$ and $\left\{M_{\omega}\right\}_{\omega \in \mathbf{R}}$. That is, we will need the fact that

$$
\forall f \in L^{2}(\mathbf{R}), \quad \lim _{x \rightarrow 0}\left\|T_{x} f-f\right\|_{2}=0=\lim _{\omega \rightarrow 0}\left\|M_{\omega} f-f\right\|_{2} .
$$

The next theorem is stated in [69]. A proof of part (a) is given there, and will not be repeated here. The proof of part (b) is similar, but since it is not given in [69] and is slightly more complicated than part (a), we prove it here.

Theorem 9.20. Assume that $g \in L^{2}(\mathbf{R})$ and $\Lambda=\left\{\left(\alpha_{k}, \beta_{k}\right)\right\}_{k=1}^{N}$ are such that $\mathcal{G}(g, \Lambda)$ is linearly independent. Then the following statements hold.

(a) There exists $\varepsilon>0$ such that $\mathcal{G}(h, \Lambda)$ is independent for any $h \in L^{2}(\mathbf{R})$ with $\|g-h\|_{2}<\varepsilon$.

(b) There exists $\varepsilon>0$ such that $\mathcal{G}\left(g, \Lambda^{\prime}\right)$ is independent for any set $\Lambda^{\prime}=$ $\left\{\left(\alpha_{k}^{\prime}, \beta_{k}^{\prime}\right)\right\}_{k=1}^{N}$ such that $\left|\alpha_{k}-\alpha_{k}^{\prime}\right|, \quad\left|\beta_{k}-\beta_{k}^{\prime}\right|<\varepsilon$ for $k=1, \ldots, N$.

Proof. (b) Let $A, B$ be frame bounds for $\mathcal{G}(g, \Lambda)$ as a frame for its span. Fix $0<\delta<A^{1 / 2} /\left(2 N^{1 / 2}\right)$. Then by (9.19), we can choose $\varepsilon$ small enough that

$$
|r| \leq \varepsilon \quad \Longrightarrow \quad\left\|T_{r} g-g\right\|_{2} \leq \delta,\left\|M_{r} g-g\right\|_{2} \leq \delta .
$$

Now suppose that $\left|\alpha_{k}-\alpha_{k}^{\prime}\right|<\varepsilon$ and $\left|\beta_{k}-\beta_{k}^{\prime}\right|<\varepsilon$ for $k=1, \ldots, N$. Then for any scalars $c_{1}, \ldots, c_{N}$ we have

$$
\begin{aligned}
\| \sum_{k=1}^{N} c_{k} & M_{\beta_{k}} T_{\alpha_{k}} g \|_{2} \\
\leq \| & \sum_{k=1}^{N} c_{k} M_{\beta_{k}}\left(T_{\alpha_{k}} h-T_{\alpha_{k}^{\prime}}\right) g \|_{2} \\
& +\left\|\sum_{k=1}^{N} c_{k}\left(M_{\beta_{k}}-M_{\beta_{k}^{\prime}}\right) T_{\alpha_{k}^{\prime}} g\right\|_{2}+\left\|\sum_{k=1}^{N} c_{k} M_{\beta_{k}^{\prime}} T_{\alpha_{k}^{\prime}} g\right\|_{2}
\end{aligned}
$$




$$
\begin{aligned}
& \leq \sum_{k=1}^{N}\left|c_{k}\right|\left\|T_{\alpha_{k}} g-T_{\alpha_{k}^{\prime}} g\right\|_{2}+\sum_{k=1}^{N}\left|c_{k}\right|\left\|\left(M_{\beta_{k}}-M_{\beta_{k}^{\prime}}\right) T_{\alpha_{k}^{\prime}} g\right\|_{2} \\
& \quad+\left\|\sum_{k=1}^{N} c_{k} M_{\beta_{k}^{\prime}} T_{\alpha_{k}^{\prime}} g\right\|_{2} \\
& =\sum_{k=1}^{N}\left|c_{k}\right|\left\|T_{\alpha_{k}-\alpha_{k}^{\prime}} g-g\right\|_{2}+\sum_{k=1}^{N}\left|c_{k}\right|\left\|M_{\beta_{k}-\beta_{k}^{\prime}} g-g\right\|_{2} \\
& \quad+\left\|\sum_{k=1}^{N} c_{k} M_{\beta_{k}^{\prime}} T_{\alpha_{k}^{\prime}} g\right\|_{2} \\
& \leq 2 \delta \sum_{k=1}^{N}\left|c_{k}\right|+\left\|\sum_{k=1}^{N} c_{k} M_{\beta_{k}^{\prime}} T_{\alpha_{k}^{\prime}} g\right\|_{2} \\
& \leq 2 \delta N^{1 / 2}\left(\sum_{k=1}^{N}\left|c_{k}\right|^{2}\right)^{1 / 2}+\left\|\sum_{k=1}^{N} c_{k} M_{\beta_{k}^{\prime}} T_{\alpha_{k}^{\prime}} g\right\|_{2} .
\end{aligned}
$$

However, we also have by Lemma 9.19 that

$$
A^{1 / 2}\left(\sum_{k=1}^{N}\left|c_{k}\right|^{2}\right)^{1 / 2} \leq\left\|\sum_{k=1}^{N} c_{k} M_{\beta_{k}} T_{\alpha_{k}} g\right\|_{2} .
$$

Combining and rearranging these inequalities, we find that

$$
\left(A^{1 / 2}-2 \delta N^{1 / 2}\right)\left(\sum_{k=1}^{N}\left|c_{k}\right|^{2}\right)^{1 / 2} \leq\left\|\sum_{k=1}^{N} c_{k} M_{\beta_{k}^{\prime}} T_{\alpha_{k}^{\prime}} g\right\|_{2} .
$$

Since $A^{1 / 2}-2 \delta N^{1 / 2}>0$, it follows that if $\sum_{k=1}^{N} c_{k} M_{\beta_{k}^{\prime}} T_{\alpha_{k}^{\prime}} g=0$ a.e. then $c_{1}=\cdots=c_{N}=0$.

Unfortunately, Theorem 9.20 cannot be combined with the known special cases, such as for compactly supported $g$ or for lattice $\Lambda$, to give a proof of the full HRT Conjecture. The problem is that $\varepsilon$ in Theorem 9.20 depends on $g$ and $\Lambda$. Analogously, a union of arbitrary open intervals $\left(r-\varepsilon_{r}, r+\varepsilon_{r}\right)$ centered at rationals $r \in \mathbf{Q}$ need not cover the entire line (consider $\varepsilon_{r}=|r-\sqrt{2}|$ ). What is needed is specific knowledge of how the value of $\varepsilon$ depends on $g$ and $\Lambda$. The proof above shows that the value of $\varepsilon$ is connected to the lower frame bound for $\mathcal{G}(g, \Lambda)$ considered as a frame for its finite span in $L^{2}(\mathbf{R})$. This leads us to close this survey with the following fundamental problem.

- Given $g \in L^{2}(\mathbf{R})$ and a finite set $\Lambda \subset \mathbf{R}^{2}$, find explicit values for the frame bounds of $\mathcal{G}(g, \Lambda)$ as a frame for its span.

Explicit here means that the frame bounds should be expressed in some computable way as a function of $g$ (or its properties, such as the size of its 
support) and the points in $\Lambda$. This is clearly an important problem with practical implications, since implementations will always involve finite sets. Surprisingly little is known regarding such frame bounds; the best results appear to be those of Christensen and Lindner [34]. In fact, even the seemingly "simpler" problem of computing explicit frame bounds for finite sets of exponentials $\left\{e^{2 \pi i \alpha_{k} x}\right\}_{k=1}^{N}$ as frames for their spans in $L^{2}[0,1]$ is very difficult, but still of strong interest, see [33].

\section{Acknowledgments}

Deep and heartfelt thanks go to John Benedetto for introducing me to harmonic analysis, wavelets, and time-frequency analysis, and for his constant friendship and support. Thanks also to Jay Ramanathan and Pankaj Topiwala for their collaboration that led to our conjecture and partial results, to Palle Jorgensen for suggestions and advice on that initial paper, to Gestur Ólafsson and Larry Baggett for their interest in the conjecture and for encouraging me to write the notes that became this chapter, and to the many people with whom I have discussed this conjecture, including Radu Balan, Nick Brönn, Pete Casazza, Ole Christensen, Baiqiao Deng, Hans Feichtinger, Vera Furst, Karlheinz Gröchenig, Guido Janssen, Norbert Kaiblinger, Werner Kozek, Gitta Kutyniok, Demetrio Labate, David Larson, Chris Lennard, Peter Linnell, Yura Lyubarskii, Joseph Rosenblatt, Ziemowit Rzeszotnik, Andrew Stimpson, David Walnut, and Georg Zimmermann. The support of NSF grant DMS-0139261 is also gratefully acknowledged.

\section{References}

1. A. Aldroubi, C. A. Cabrelli, and U. M. Molter, Wavelets on irregular grids with arbitrary dilation matrices and frame atoms for $L^{2}\left(\mathbb{R}^{d}\right)$, Appl. Comput. Harmon. Anal., 17 (2004), pp. 119-140.

2. A. Aldroubi and K. Gröchenig, Nonuniform sampling and reconstruction in shift-invariant spaces, SIAM Review, 43 (2001), pp. 585-620.

3. K. I. Babenko, On conjugate functions (Russian), Doklady Akad. Nauk SSSR (N. S.), 62 (1948), pp. 157-160.

4. L. Baggett, Processing a radar signal and representations of the discrete Heisenberg group, Colloq. Math., 60/61 (1990), pp. 195-203.

5. L. Baggett, H. Medina, and K. Merrill, Generalized multiresolution analyses, and a construction procedure for all wavelet sets in $\mathbf{R}^{n}$, J. Fourier Anal. Appl., 6 (1999), pp. 563-573.

6. R. Balan, P. G. Casazza, C. Heil, and Z. Landau, Deficits and excesses of frames, Adv. Comput. Math., 18 (2003), pp. 93-116.

7. R. Balan, P. G. Casazza, C. Heil, and Z. Landau, Excesses of Gabor frames, Appl. Comput. Harmon. Anal., 14 (2003), pp. 87-106.

8. R. Balan, P. G. Casazza, C. Heil, and Z. Landau, Density, overcompleteness, and localization of frames, I. Theory, preprint (2005). 
9. R. Balan, P. G. Casazza, C. Heil, and Z. Landau, Density, overcompleteness, and localization of frames, II. Gabor frames, preprint (2005).

10. R. Balian, Un principe d'incertitude fort en théorie du signal ou en mécanique quantique, C. R. Acad. Sci. Paris, 292 (1981), pp. 1357-1362.

11. G. Battle, Heisenberg proof of the Balian-Low theorem, Lett. Math. Phys., 15 (1988), pp. 175-177.

12. J. J. Benedetto, Gabor representations and wavelets, in: Commutative Harmonic Analysis (Canton, NY, 1987), Contemp. Math., Vol. 91, Amer. Math. Soc., Providence, RI, 1989, pp. 9-27.

13. J. J. Benedetto, Harmonic Analysis and Applications, CRC Press, Boca Raton, FL, 1997.

14. J. J. Benedetto, W. Czaja, P. Gadziński, and A. M. Powell, The Balian-Low Theorem and regularity of Gabor systems, J. Geom. Anal., 13 (2003), pp. 217232.

15. J. J. Benedetto, W. Czaja, and A. Ya. Maltsev, The Balian-Low theorem for the symplectic form on $\mathbf{R}^{2 d}$, J. Math. Phys., 44 (2003), pp. 1735-1750.

16. J. J. Benedetto and M. Fickus, Finite normalized tight frames, Adv. Comput. Math., 18 (2003), pp. 357-385.

17. J. J. Benedetto, C. Heil, and D. F. Walnut, Differentiation and the Balian-Low Theorem, J. Fourier Anal. Appl., 1 (1995), pp. 355-402.

18. J. J. Benedetto and M. Leon, The construction of single wavelets in $D$ dimensions, J. Geom. Anal., 11 (2001), pp. 1-15.

19. A. Boggess and F. J. Narcowich, A First Course in Wavelets with Fourier Analysis, Prentice-Hall, Upper Saddle River, NJ, 2001.

20. M. Bownik and Z. Rzeszotnik, The spectral function of shift-invariant spaces, Michigan Math. J., 51 (2003), pp. 387-414.

21. C. A. Cabrelli, C. Heil, and U. M. Molter, Self-similarity and multiwavelets in higher dimensions, Memoirs Amer. Math. Soc., 170, No. 807 (2004).

22. P. G. Casazza, The art of frame theory, Taiwanese J. Math., 4 (2000), pp. 129 201.

23. P. G. Casazza, O. Christensen, A. M. Lindner, and R. Vershynin, Frames and the Feichtinger conjecture, Proc. Amer. Math. Soc., 133 (2005), pp. 1025-1033.

24. P. G. Casazza, M. Fickus, J. Kovačević, M. T. Leon, and J. C. Tremain, A physical interpretation of tight frames, Chapter 4, this volume (2006).

25. P. G. Casazza and N. J. Kalton, Roots of complex polynomials and WeylHeisenberg frame sets, Proc. Amer. Math. Soc., 130 (2002), pp. 2313-2318.

26. P. G. Casazza and J. C. Tremain, The Kadison-Singer problem in mathematics and engineering, Proc. Natl. Acad. Sci. USA, 103 (2006), pp. 2032-2039.

27. S. Chen, D. Donoho, and M. A. Saunders, Atomic decomposition by basis pursuit, SIAM Review, 43 (2001), pp. 129-157.

28. O. Christensen, An Introduction to Frames and Riesz Bases, Birkhäuser, Boston, 2003.

29. O. Christensen and P. G. Casazza, Frames containing a Riesz basis and preservation of this property under perturbations, SIAM J. Math. Anal., 29 (1998), pp. 266-278.

30. O. Christensen and Y. C. Eldar, Oblique dual frames and shift-invariant spaces, Appl. Comput. Harmon. Anal., 17 (2004), pp. 48-68.

31. O. Christensen and C. Heil, Perturbations of Banach frames and atomic decompositions, Math. Nachr., 185 (1997), pp. 33-47. 
32. O. Christensen, B. Deng, and C. Heil, Density of Gabor frames, Appl. Comput. Harmon. Anal., 7 (1999), pp. 292-304.

33. O. Christensen and A. M. Lindner, Frames of exponentials: lower frame bounds for finite subfamilies, and approximation of the inverse frame operator, Linear Algebra Appl., 323 (2001), pp. 117-130.

34. O. Christensen and A. M. Lindner, Lower bounds for finite wavelet and Gabor systems, Approx. Theory Appl. (N.S.), 17 (2001), pp. 18-29.

35. O. Christensen and A. M. Lindner, Decompositions of wavelets and Riesz frames into a finite number of linearly independent sets, Linear Algebra Appl., 355 (2002), pp. 147-159.

36. E. Cordero and K. Gröchenig, Localization of frames. II, Appl. Comput. Harmon. Anal., 17 (2004), pp. 29-47.

37. W. Czaja, G. Kutyniok, and D. Speegle, Geometry of sets of parameters of wave packet framess, Appl. Comput. Harmon. Anal., 20 (2006), pp. 108-125.

38. W. Czaja and A. Powell, Recent developments in the Balian-Low Theorem, Chapter 5, this volume (2006).

39. X. Dai, D. R. Larson, and D. M. Speegle, Wavelet sets in $\mathbf{R}^{n}$, J. Fourier Anal. Appl., 3 (1997), pp. 451-456.

40. I. Daubechies, The wavelet transform, time-frequency localization and signal analysis, IEEE Trans. Inform. Theory, 36 (1990), pp. 961-1005.

41. I. Daubechies, Ten Lectures on Wavelets, SIAM, Philadelphia, 1992.

42. I. Daubechies, A. Grossmann, and Y. Meyer, Painless nonorthogonal expansions, J. Math. Phys., 27 (1986), pp. 1271-1283.

43. I. Daubechies and A. J. E. M. Janssen, Two theorems on lattice expansions, IEEE Trans. Inform. Theory, 39 (1993), pp. 3-6.

44. I. Daubechies, H. Landau, and Z. Landau, Gabor time-frequency lattices and the Wexler-Raz identity, J. Fourier Anal. Appl., 1 (1995), pp. 437-478.

45. B. Deng and C. Heil, Density of Gabor Schauder bases, in: Wavelet Applications in Signal and Image Processing VIII (San Diego, CA, 2000), A. Aldroubi, A. Lane, and M. Unser, eds., Proc. SPIE Vol. 4119, SPIE, Bellingham, WA, 2000, pp. 153-164.

46. R. J. Duffin and A. C. Schaeffer, A class of nonharmonic Fourier series, Trans. Amer. Math. Soc., 72 (1952), pp. 341-366.

47. K. Dykema, D. Freeman, K. Kornelson, D. Larson, M. Ordower, and E. Weber, Ellipsoidal tight frames and projection decomposition of operators, Illinois $J$. Math., 48 (2004), pp. 477-489.

48. G. Edgar and J. Rosenblatt, Difference equations over locally compact abelian groups, Trans. Amer. Math. Soc., 253 (1979), pp. 273-289.

49. G. B. Folland, Harmonic Analysis on Phase Space, Princeton Univ. Press, Princeton, NJ, 1989.

50. G. B. Folland and A. Sitaram, The uncertainty principle: A mathematical survey, J. Fourier Anal. Appl., 3 (1997), pp. 207-238.

51. D. Gabor, Theory of communication, J. Inst. Elec. Eng. (London), 93 (1946), pp. 429-457.

52. J.-P. Gabardo and D. Han, Aspects of Gabor analysis and operator algebras, in: Advances in Gabor Analysis, H. G. Feichtinger and T. Strohmer, eds., Birkhäuser, Boston, 2003, pp. 153-169.

53. J.-P. Gabardo and D. Han, Balian-Low phenomenon for subspace Gabor frames, J. Math. Phys., 45 (2004), pp. 3362-3378. 
54. B. R. Gelbaum, Notes on Banach spaces and bases, An. Acad. Brasil. Ci., 30 (1958), pp. 29-36.

55. K. Gröchenig, Foundations of Time-Frequency Analysis, Birkhäuser, Boston, 2001.

56. K. Gröchenig, Uncertainty principles for time-frequency representations, in: Advances in Gabor Analysis, H. G. Feichtinger and T. Strohmer, eds., Birkhäuser, Boston, 2003, pp. 11-30.

57. K. Gröchenig, Localized frames are finite unions of Riesz sequences, Adv. Comput. Math., 18 (2003), pp. 149-157.

58. K. Gröchenig, Localization of frames, Banach frames, and the invertibility of the frame operator, J. Fourier Anal. Appl., 10 (2004), pp. 105-132.

59. K. Gröchenig, D. Han, C. Heil, and G. Kutyniok, The Balian-Low Theorem for symplectic lattices in higher dimensions, Appl. Comput. Harmon. Anal., 13 (2002), pp. 169-176.

60. K. Gröchenig and H. Razafinjatovo, On Landau's necessary density conditions for sampling and interpolation of band-limited functions, J. London Math. Soc. (2), 54 (1996), pp. 557-565.

61. D. Han and D. R. Larson, Frames, bases and group representations Memoirs Amer. Math. Soc., 147, No. 697 (2000).

62. E. Hayashi, S. Li, and T. Sorrells, Gabor duality characterizations, Chapter 7, this volume (2006)

63. C. Heil, Wiener Amalgam Spaces in Generalized Harmonic Analysis and Wavelet Theory, Ph.D. Thesis, University of Maryland, College Park, MD, 1990.

64. C. Heil, A Basis Theory Primer, manuscript, 1997. Electronic version available at http://www.math.gatech.edu/ heil.

65. C. Heil, An introduction to weighted Wiener amalgams, in: Wavelets and their Applications (Chennai, January 2002), M. Krishna, R. Radha and S. Thangavelu, eds., Allied Publishers, New Delhi, 2003, pp. 183-216.

66. C. Heil, Integral operators, pseudodifferential operators, and Gabor frames, in: Advances in Gabor Analysis, H. G. Feichtinger and T. Strohmer, eds., Birkhäuser, Boston, 2003, pp. 153-169.

67. C. Heil and G. Kutyniok, Density of weighted wavelet frames, J. Geom. Anal., 13 (2003), pp. 479-493.

68. C. Heil and G. Kutyniok, The Homogeneous Approximation Property for wavelet frames, preprint (2005).

69. C. Heil, J. Ramanathan, and P. Topiwala, Linear independence of timefrequency translates, Proc. Amer. Math. Soc., 124 (1996), pp. 2787-2795.

70. C. Heil, J. Ramanathan, and P. Topiwala, Singular values of compact pseudodifferential operators, J. Funct. Anal., 150 (1996), pp. 426-452.

71. C. E. Heil and D. F. Walnut, Continuous and discrete wavelet transforms, SIAM Review, 31 (1989), pp. 628-666.

72. C. Heil and D. F. Walnut, eds., Fundamental Papers in Wavelet Theory, Princeton University Press, Princeton, NJ, 2006.

73. E. Hernández, D. Labate, and G. Weiss, A unified characterization of reproducing systems generated by a finite family, II, J. Geom. Anal., 12 (2002), pp. 615-662.

74. E. Hernández and G. Weiss, A First Course on Wavelets, CRC Press, Boca Raton, FL, 1996. 
75. G. Higman, The units of group-rings, Proc. London Math. Soc. (2), 46 (1940), pp. 231-248.

76. J. R. Holub, Pre-frame operators, Besselian frames, and near-Riesz bases in Hilbert spaces, Proc. Amer. Math. Soc., 122 (1994), pp. 779-785.

77. A. J. E. M. Janssen, Bargmann transform, Zak transform, and coherent states, J. Math. Phys., 23 (1982), pp. 720-731.

78. A. J. E. M. Janssen, The Zak transform: a signal transform for sampled timecontinuous signals, Philips J. Res., 43 (1988), pp. 23-69.

79. A. J. E. M. Janssen, Signal analytic proofs of two basic results on lattice expansions, Appl. Comput. Harmon. Anal., 1 (1994), pp. 350-354.

80. A. J. E. M. Janssen, Duality and biorthogonality for Weyl-Heisenberg frames, J. Fourier Anal. Appl., 1 (1995), pp. 403-436.

81. A. J. E. M. Janssen, Representations of Gabor frame operators, in: Twentieth Century Harmonic Analysis - A Celebration (Il Ciocco, 2000), NATO Sci. Ser. II Math. Phys. Chem., 33, Kluwer Acad. Publ., Dordrecht, 2001, pp. 73-101.

82. A. J. E. M. Janssen, Zak transforms with few zeros and the tie, in: Advances in Gabor Analysis, H. G. Feichtinger and T. Strohmer, eds., Birkhäuser, Boston, 2003, pp. 31-70.

83. A. J. E. M. Janssen, On generating tight Gabor frames at critical density, J. Fourier Anal. Appl., 9 (2003), pp. 175-214.

84. A. J. E. M. Janssen and T. Strohmer, Hyperbolic secants yield Gabor frames, Appl. Comput. Harmon. Anal., 12 (2002), pp. 259-267.

85. R. Kadison and I. Singer, Extensions of pure states, Amer. J. Math., 81 (1959), pp. 383-400.

86. K. S. Kazarian, F. Soria, and R. E. Zink, On rearranges orthogonal systems as quasibases in weighted $L^{p}$ spaces, in: Interaction between Functional Analysis, Harmonic Analysis, and Probability (Columbia, MO, 1994), N. Kalton, E. Saab, and S. Montgomery-Smith, eds., Lecture Notes in Pure and Appl. Math. Vol. 175, Dekker, New York, 1996, pp. 239-247,

87. H. O. Kim and J. K. Lim, New characterizations of Riesz bases, Appl. Comput. Harmon. Anal., 4 (1997), pp. 222-229.

88. G. Kutyniok, Linear independence of time-frequency shifts under a generalized Schrödinger representation, Arch. Math. (Basel), 78 (2002), pp. 135-144.

89. G. Kutyniok, Computation of the density of weighted wavelet systems, in: Wavelets: Applications in Signal and Image Processing, M. Unser, A. Aldroubi, and A. Laine eds., SPIE Proc. Vol. 5207, SPIE, San Diego, 2003, pp. 393-404.

90. D. Labate, A unified characterization of reproducing systems generated by a finite family, J. Geom. Anal., 12 (2002), pp. 469-491.

91. H. Landau, On the density of phase-space expansions, IEEE Trans. Inform. Theory, 39 (1993), pp. 1152-1156.

92. S. Li and H. Ogawa, Pseudo-duals of frames with applications, Appl. Comput. Harmon. Anal., 11 (2001), 289-304.

93. J. Lindenstrauss and L. Tzafriri, Classical Banach Spaces, I, Springer-Verlag, New York, 1977.

94. P. A. Linnell, Zero divisors and $L^{2}(G)$, C. R. Acad. Sci. Paris Sér. I Math., 315 (1992), pp. 49-53.

95. P. A. Linnell, Analytic versions of the zero divisor conjecture, in: Geometry and Cohomology in Group Theory, P. H. Kropholler, G. A. Niblo, and R. Stöhr, eds., London Math. Soc. Lecture Note Series, Cambridge University Press, Cambridge, 1998, pp. 209-248. 
96. P. A. Linnell, Von Neumann algebras and linear independence of translates, Proc. Amer. Math. Soc., 127 (1999), pp. 3269-3277.

97. F. Low, Complete sets of wave packets, in: A Passion for Physics-Essays in Honor of Geoffrey Chew, C. DeTar, J. Finkelstein, and C. I. Tan, eds., World Scientific, Singapore, 1985, pp. 17-22.

98. J. Marti, Introduction to the Theory of Bases, Springer-Verlag, New York, 1969.

99. A. Olevskii, Completeness in $L^{2}(\mathbf{R})$ of almost integer translates, C. R. Acad. Sci. Paris, 324 (1997), pp. 987-991.

100. A. Olevskii and A. Ulanovskii, Almost integer translates. Do nice generators exist?, J. Fourier Anal. Appl., 10 (2004), pp. 93-104.

101. T. E. Olson and R. A. Zalik, Nonexistence of a Riesz basis of translates, in: Approximation Theory, Lecture Notes in Pure and Applied Math., Vol. 138, Dekker, New York, 1992, pp. 401-408.

102. A. M. Perelomov, On the completeness of a system of coherent states (English translation), Theoret. Math. Phys., 6 (1971), pp. 156-164.

103. J. Ramanathan and T. Steger, Incompleteness of sparse coherent states, Appl. Comput. Harmon. Anal., 2 (1995), pp. 148-153.

104. M. Rieffel, Von Neumann algebras associated with pairs of lattices in Lie groups, Math. Ann., 257 (1981), pp. 403-418.

105. J. Rosenblatt, Linear independence of translations, J. Austral. Math. Soc. (Series A), 59 (1995), pp. 131-133.

106. Z. Rzeszotnik, private communication, 2004.

107. K. Seip, On the connection between exponential bases and certain related sequences in $L^{2}(-\pi, \pi)$, J. Funct. Anal., 130 (1995), pp. 131-160.

108. I. Singer, Bases in Banach Spaces I, Springer-Verlag, New York, 1970.

109. T. Strohmer and R. W. Heath, Jr., Grassmannian frames with applications to coding and communication, Appl. Comput. Harmon. Anal., 14 (2003), pp. 257275 .

110. W. Sun and X. Zhou, Density and stability of wavelet frames, Appl. Comput. Harmon. Anal., 15 (2003), pp. 117-133.

111. D. F. Walnut, An Introduction to Wavelet Analysis, Birkhäuser, Boston, 2002.

112. P. Walters, An Introduction to Ergodic Theory, Springer-Verlag, New York, 1982.

113. Y. Wang, Sparse complete Gabor systems on a lattice, Appl. Comput. Harmon. Anal., 16 (2004), pp. 60-67.

114. R. Young, An Introduction to Nonharmonic Fourier Series, Revised First Edition, Academic Press, San Diego, 2001.

115. R. A. Zalik, On approximation by shifts and a theorem of Wiener, Trans. Amer. Math. Soc., 243 (1978), pp. 299-308.

116. R. A. Zalik, On fundamental sequences of translates, Proc. Amer. Math. Soc., 79 (1980), pp. 255-259. 